\title{
Secure Communications for the Two-user Broadcast Channel with Random Traffic
}

Parthajit Mohapatra, Nikolaos Pappas, J emin Lee, Tony Q.S. Quek and Vangelis Angelakis

The self-archived postprint version of this journal article is available at Linköping University Institutional Repository (DiVA):

http:// urn.kb.se/ resolve?urn=urn:nbn:se:liu:diva-146030

N.B.: When citing this work, cite the original publication.

Mohapatra, P., Pappas, N., Lee, J., Quek, T. Q., Angelakis, V., (2018), Secure Communications for the Two-user Broadcast Channel with Random Traffic, IEEE Transactions on Information Forensics and Security, 13(9), 2294-2309. https:// doi.org/ 10.1109/TIFS.2018.2818076

Original publication available at:

https:// doi.org/ 10.1109/TIFS.2018.2818076

Copyright: Institute of Electrical and Electronics Engineers (IEEE) http:// www.ieee.org/ index.html

(c)2018 IEEE. Personal use of this material is permitted. However, permission to reprint/republish this material for advertising or promotional purposes or for creating new collective works for resale or redistribution to servers or lists, or to reuse any copyrighted component of this work in other works must be obtained from the IEEE. 


\title{
Secure Communications for the Two-user Broadcast Channel with Random Traffic
}

\author{
Parthajit Mohapatra, Member, IEEE, Nikolaos Pappas, Member, IEEE, \\ Jemin Lee, Member, IEEE, Tony Q. S. Quek, Fellow, IEEE, \\ and Vangelis Angelakis, Senior Member, IEEE
}

\begin{abstract}
In this work, we study the stability region of the two-user broadcast channel (BC) with bursty data arrivals and security constraints. It is assumed that one of the receivers has a secrecy constraint, i.e., its packets need to be kept secret from the other receiver, which is defined based on SINR. The receiver with secrecy constraint has full-duplex capability to send a jamming signal for improving its service rate. The stability region of the two-user BC with secrecy constraint is characterized for the general decoding case. Then, assuming two different decoding schemes the respective stability regions are derived. The fullduplex operation of receiver results in self-interference, and the effect of imperfect self-interference cancelation on the stability region is also investigated. The stability region of the $\mathrm{BC}$ with a secrecy constraint, where the receivers do not have full duplex capability can be obtained as a special case of the results derived in this paper. In addition, the paper considers the problem of maximizing the saturated throughput of the queue for which there is no secrecy constraint under minimum service guarantees for the other queue. The results provide new insights on the effect of the secrecy constraint on the stability region of the BC. It is found that the stability region with secrecy constraint is sensitive to the degree of self-interference cancelation.
\end{abstract}

\section{INTRODUCTION}

In the last decade, physical layer secrecy has emerged as a promising approach for security in wireless communications. The physical layer techniques inspired from the information theoretic results often require infinitely backlogged users, i.e., users always have data to transmit. However, such an assumption does not capture the bursty nature of the sources. To account for the bursty nature of the sources, the notion of stability region [2] has been introduced. In many communication scenarios, it is required to serve multiple users simultaneously and the data arrival at the transmitter is bursty

Parthajit Mohapatra is with the G. S. Sanyal School of Telecommunications, Indian Institute of Technology, Kharagpur, India (e-mail: parthajit@gssst.iitkgp.ernet.in).

Nikolaos Pappas and Vangelis Angelakis are with the Department of Science and Technology, Linköping University, Norrköping SE-60174, Sweden (e-mail: \{nikolaos.pappas, vangelis.angelakis\}@liu.se).

Jemin Lee is with the Department of Information and Communication Engineering, Daegu Gyeongbuk Institute of Science and Technology (DGIST), Korea (e-mail: jmnlee@dgist.ac.kr).

Tony Q. S. Quek is with the Information Systems Technology and Design Pillar, Singapore University of Technology and Design, Singapore (e-mail: tonyquek@sutd.edu.sg).

This work was presented in part in IEEE ICC 2016 [1].

The corresponding author is J. Lee.

This work was supported in part by research grants from the National Research Foundation of Korea (NRF) grant funded by the Korea government (MSIP) (No. 2017R1C1B2009280), the DGIST R\&D Program of the Ministry of Science and ICT (17-ST-02), ELLIIT, and CENIIT. in nature. In addition, users may have different levels of security requirements. To examine jointly these two important aspects of communication, we consider a two-user broadcast channel (BC) [3] with bursty packet arrivals, where the packets of one of the queues need to be kept secret from the unintended receiver. We further assume that the receiver with the secrecy constraint has full-duplex capability. Thus, the full-duplex receiver can send a jamming signal to impede the decoding of its packets by the other receiver. The effect of the secrecy constraint on the stability region is not yet well understood. Furthermore, the stability of the queues can be affected due to the secrecy constraint and the self-interference caused because of the simultaneous transmission and reception at the receiver. Hence, the analysis of this model can provide useful insights on the system performance as well as help to understand the effect of bursty nature of sources in a broadcast channel with secrecy constraint.

\section{A. Related Work}

The capacity of the $\mathrm{BC}$ has been analyzed extensively with and without secrecy constraints in the existing literature [3][7]. However, the capacity region of the general $\mathrm{BC}$ is still unknown even without a secrecy constraint at the receiver. The achievable rate region derived in [6] is the best known achievable rate region for a general discrete memoryless BC. The work in [5] provided a partial characterization of the capacity region of the two-user Gaussian fading BC.

Exploiting randomness in a physical channel to ensure secrecy was first considered for the wiretap channel, where the legitimate transmitter needs to send a message to the legitimate receiver securely in the presence of an eavesdropper [8]. It was shown that secure communication is possible between the legitimate users without using a secret key between the legitimate nodes. The result of the wiretap channel was generalized in [6] for the BC, where one of the messages needs to be kept secret from the unintended receiver. The effect of user cooperation on the secrecy capacity of the $\mathrm{BC}$ has been investigated in [7]. The problem of secure broadcasting over fading channel has been considered in [9]. The results related to other multiuser scenarios with secrecy constraints can be found in [10]-[12].

In wireless networks, the arrivals of data at the transmitter are random and the vast majority of the works in information theory have the assumption of backlogged users [6]-[8], [10][13]. When the traffic is bursty in nature, the stability region 
or stable throughput region becomes an appropriate measure of rates in packets/slot in wireless networks [14]. The stability region is defined as the set of all arrival rates for which all queues inside the network are stable, i.e., the length of each queue is finite [2]. The work in [15] provided a theoretical treatment of some basic problems related to stability of the broadcast channel. The stability region of the two-user BC without secrecy constraint has been explored in [16], [17]. The stability region has been studied for several other communication models, such as the two-user interference channel [18]. The primary difficulty in the derivation of the stability region is the interaction among the queues, and to overcome this difficulty, stochastic dominance [2] was used. However, the characterization of the stability region of multi-users systems (where the number of users is more than 2) is a challenging problem and a detailed discussion in this regard can be found in [19].

The stability region has been analyzed for multiple users scenarios with secrecy constraints [20]-[22]. In [20], stability conditions are obtained for a slotted ALOHA network with secrecy constraints. In this case, all users who are not transmitting are the eavesdroppers. A wireless broadcast network model with secrecy constraints is investigated in [21], [22], where a source node broadcasts confidential message to user nodes, where each message is required to be decoded by the intended user and to be kept secret from all other users. In [21], [22], secrecy, reliability, and stability are jointly considered for network utility maximization.

\section{B. Contributions}

In this paper, the stable throughput region of the $\mathrm{BC}$ with a secrecy constraint and under bursty traffic is analyzed. It is assumed that the receiver for which packets need to be kept secret is assumed to have full-duplex capability. To the best of authors' knowledge, the stability region with different decoding schemes and secrecy constraint has not been analyzed in literature and the results presented in this paper with different decoding schemes give a unified view on the effect of secrecy constraint and imperfect self-interference cancelation on the stability region of the BC. The transmitter has two queues and the packets sent from the first and the second queues are for receiver 1 and receiver 2 , respectively. The packets for receiver 1 need to be kept secret from receiver 2 . In practice, such scenario can arise in a cellular network, where users have different subscription options, which produces different levels of data secrecy for users, i.e., either a certain data is required to be kept confidential or non-confidential. Another practical scenario can appear in IoT networks where there are sensors collecting data from the environment. The collected data can be categorized to non-confidential ones (e.g., outdoors temperature) or confidential ones (e.g., a baby monitor feed). The data collecting node can store the confidential data and the non-confidential to different queues. The data are transmitted through a wireless channel to two different receivers, the first one requests the confidential data while the second receiver requests non-confidential data.

To improve the stable throughput of the first queue, the receiver 1 has full duplex capability and it can generate jamming signals to receiver 2 while receiving packets from the transmitter. Sending jamming signal to increase the performance of the system with secrecy constraint has been explored in the existing literature [7], [23]-[25]. However, to the best of authors' knowledge, the role of full-duplex receiver with imperfect self-interference cancelation on the stability region with secrecy constraint has not been explored in the existing literature. In this case, the derivation of the stability region is challenging since there is a correlation between the queues, and the reliability and security criteria need to be taken into account jointly. Furthermore, in comparison to the works in [21], [22], which assume all the nodes know the channel state information, we assume that channel state information is not available at the transmitter. The transmitter only knows the statistics of the channels. The secrecy performance over wireless fading channel is often characterized by either ergodic secrecy capacity or secrecy outage probability, which do not give an insight on eavesdropper's ability to decode the secret data [26]. In this work, we have considered SINR based secrecy metric, which takes the eavesdropper's ability to decode the confidential data. ${ }^{1}$

The main contributions of this work can be summarized as follows.

1) The stability region of the two-user BC with secrecy constraint is obtained by considering different decoding capabilities at the receivers. Specifically, we consider the case of decoding by treating interference as noise (i.e., limited decoding capability case) and performing successive decoding (i.e., high decoding capability case) for each of the receivers. The stability regions are derived using the stochastic dominance technique after determining the success probabilities with and without secrecy constraints for the different decoding schemes. The derivation of the success probability is nontrivial as it is required to satisfy the secrecy and reliability criteria at the receiver.

2) The self-interference cancelation capability at receiver 1 is also taken into account in the analysis of the stability region. Although receiver 1 transmits a jamming signal to receiver 2 to enhance the data confidentiality, it can also degrade its own throughput due to the selfinterference. We explore how the self-interference cancelation capability affects the stability region. Furthermore, the stability region of the $\mathrm{BC}$ without full-duplex capability at receiver 1 can be obtained as a special case of the results derived in this paper by setting the jamming power to zero.

3) The results derived in this paper are also useful to analyze other communication scenarios such as queues with different congestion levels. One such problem considered in this paper is to maximize the saturated throughput of the queue, which does not have secrecy constraint, while guaranteeing the minimum service rate for the other queue.

In comparison to our preliminary work presented in [1], the

\footnotetext{
${ }^{1}$ For more detailed discussion on various secrecy metrics for physical layer secrecy, one can refer to [26], [27].
} 


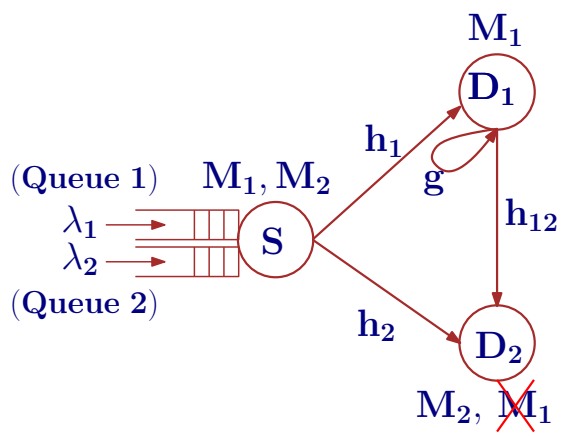

Fig. 1. The two-user broadcast channel with the security constraint: receiver 1 has full-duplex capability.

following additional results have been derived in this paper. In [1], it was assumed that receiver 2 cannot perform successive decoding. Here, the stability region is also derived when receiver 2 can perform successive decoding and receiver 1 treats interference as noise while decoding its intended packet. The derivation also takes account of the fact that if receiver 2 fails to decode the packet of first user in the successive decoding, then it attempts to decode the packet of the other user by treating interference as noise. Using the results derived under different decoding assumptions, the closures of the stability regions are obtained for two cases: (a) fixed power at the transmitter, and (b) fixed jamming power at receiver 1. Beside these, the problem of optimizing jamming power for maximizing the saturated throughput of second receiver while guaranteeing a required service rate for the first user is also explored.

\section{SYSTEM MODEL}

We consider a two-user broadcast channel (BC) as shown in Fig. 1, where a single transmitter has two different queues. The $i^{\text {th }}(i=1,2)$ queue contains the packets intended to receiver $i$ (denoted by $D_{i}, i=1,2$ ). There is no secrecy constraint for the packets sent from the second queue, but the packets sent from the first queue are required to be kept secret from the second receiver, i.e., $D_{2}$. To enhance the communication secrecy, $D_{1}$ is assumed to have full-duplex capability, so it can receive the packets from the transmitter and send a jamming signal to the other receiver $\left(D_{2}\right)$, simultaneously. The jamming signal sent by $D_{1}$ can cause additional interference at $D_{2}$ and may help to increase the secrecy throughput of $D_{1}$. The system model considered in this paper can capture a scenario where one of the users requests for confidential data and another user requests for data without security requirements from the same transmitter. The signal $y_{i}^{(t)}(i \in\{1,2\})$ received at receiver $i$ in time-slot $t$ is modeled as

$$
\begin{aligned}
& y_{1}^{(t)}=h_{1}^{(t)} x^{(t)}+g x_{\mathbf{J}}^{(t)}+z_{1}^{(t)}, \\
& y_{2}^{(t)}=h_{2}^{(t)} x^{(t)}+h_{12}^{(t)} x_{\mathbf{J}}^{(t)}+z_{2}^{(t)},
\end{aligned}
$$

where $z_{i}^{(t)} \sim \mathcal{C N}(0,1)$, and $h_{i}^{(t)}$ and $h_{i j}^{(t)}$ denote Rayleigh block fading channel between the transmitter and the $i$-th receiver and that between the $i$-th receiver and the $j$-th receiver, respectively. When both queues are non-empty, the transmitter sends $x^{(t)}=x_{1}^{(t)}+x_{2}^{(t)}$, where $x_{i}^{(t)}$ is the packet intended for receiver $i$. When only the $i^{\text {th }}$ queue has packets to send, then the transmitter sends $x^{(t)}=x_{i}^{(t)}(i \in\{1,2\})$. The transmitter assigns power $P_{i}$ for messages (packets) from queue $\mathrm{i}(i \in\{1,2\})$ and receiver 1 can transmit a jamming signal with power $P_{J}$.

Here, it is assumed that all channels are independent. In (1), $g$ is the residual self-interference, which is modeled by a scalar $g \in[0,1]$. The self-interference cancelation at the fullduplex receiver is assumed to be imperfect and is modeled by residual self-interference-to-power ratio (SIPR) $g^{2}$, i.e., when the transmit power of a node is $P_{J}$, then the residual self-interference power is $g^{2} P_{J}$ [28]-[35]. The residual selfinterference channel gain $g$ needs to be characterized according to the cancelation algorithm. For instance, after a digital domain cancelation, the residual self-interference channel gain $g$ can be modeled as $g=h_{I}-\hat{h}_{I}$, where $h_{I}$ and $\hat{h}_{I}$ are the self-interfering channel and the estimate of the selfinterfering channel, respectively [28]-[33]. Then, $g^{2}$ can be modeled as a constant value such as $\sigma_{e}^{2}$ for the estimation-error variance [28], [30]-[33]. This captures the accuracy of the selfinterference cancelation technique. When $g=1$, it indicates that no self-interference cancelation technique has been used and $g=0$ indicates that perfect self-interference cancelation has been achieved. We also would like to note that once the probability distribution function (PDF) of the self-interference channel gain $(g)$ is available the analytic results can be readily extended within the framework of this paper.

The event $\mathcal{D}_{i / \mathcal{T}}$ denotes that the receiver $i$ is able to decode the packet sent from the $i^{\text {th }}$ queue of the transmitter given a set of non-empty queues $\mathcal{T}$. The event $\mathcal{D}_{i / i, \mathcal{T}}^{s}$ denotes that the user $i$ is able to decode its intended packet and the other users $j \in \mathcal{T} \backslash\{i\}$ are not able to decode the packet sent from the $i^{\text {th }}$ queue. We use $s$ as superscript in $\mathcal{D}_{i / i, \mathcal{T}}^{s}$ as it maps the event that a packet for user $i$ is confidential to other users.

The packet arrival process at the $i$-th queue is assumed to be independent and stationary with mean rate $\lambda_{i}$ in packets per slot. Both queues are assumed to have infinite capacity to store incoming packets (i.e., no loss system) and $Q_{i}$ denotes the length of the $i^{\text {th }}$ queue. If one of the queues is non-empty, then the transmitter sends a packet in a time slot. It is also assumed that acknowledgments sent by the receivers are instantaneous and error free. The average service rate for the first queue is

$$
\mu_{1}=\mathcal{P}\left(Q_{2}>0\right) \mathcal{P}\left(\mathcal{D}_{1 / 1,2}^{s}\right)+\mathcal{P}\left(Q_{2}=0\right) \mathcal{P}\left(\mathcal{D}_{1 / 1}^{s}\right) .
$$

Similarly, the average service rate for the second queue is

$$
\mu_{2}=\mathcal{P}\left(Q_{1}>0\right) \mathcal{P}\left(\mathcal{D}_{2 / 1,2}\right)+\mathcal{P}\left(Q_{1}=0\right) \mathcal{P}\left(\mathcal{D}_{2 / 2}\right) .
$$

Here, $\mathcal{P}(\mathcal{A})$ is the probability of occurrence of the event $\mathcal{A}$. Hence, $\mathcal{P}\left(\mathcal{D}_{i / \mathcal{T}}\right)$ and $\mathcal{P}\left(\mathcal{D}_{i / i, \mathcal{T}}^{s}\right)$ denote the probabilities that the receiver $D_{i}$ decodes its intended packets successfully in the absence/presence of the secrecy constraint, respectively.

\section{A. The stability region of the general case}

The following definition of queue stability is used [36]: Definition 1. Let $Q_{i}^{(t)}$ denotes the length of the queue $i$ 
at the beginning of the time-slot $t$. The queue is said to be stable if $\lim _{t \rightarrow \infty} \mathcal{P}\left(Q_{i}^{(t)}<x\right)=F(x)$ and $\lim _{x \rightarrow \infty} F(x)=1$. The queue is said to be sub-stable if $\lim _{x \rightarrow \infty} \lim _{t \rightarrow \infty} \inf \mathcal{P}\left(Q_{i}^{(t)}<x\right)=1$. If a queue is stable, then it is also sub-stable. A queue is said to be unstable if the queue is not sub-stable.

From the definition of queue stability, the stability region is defined as the set of all possible arrival rates at the queues for which the queues remain stable. The average service rates of the first and second queues are given by (2) and (3), respectively. Note that the average service rate of each queue depends on the queue length of the other queue, so it is non-trivial to determine the stability region. To overcome this difficulty, the stochastic dominance technique [2] can be applied which is based on the construction of hypothetical dominant systems. The stability region $\mathcal{R}_{1}$ obtained from the first dominant system, i.e., when the first queue transmits dummy packets, is as follows:

$$
\begin{gathered}
\mathcal{R}_{1}=\left\{\left(\lambda_{1}, \lambda_{2}\right): \frac{\lambda_{1}}{\mathcal{P}\left(\mathcal{D}_{1 / 1}^{s}\right)}+\frac{\mathcal{P}\left(\mathcal{D}_{1 / 1}^{s}\right)-\mathcal{P}\left(\mathcal{D}_{1 / 1,2}^{s}\right)}{\mathcal{P}\left(\mathcal{D}_{1 / 1}^{s}\right) \mathcal{P}\left(\mathcal{D}_{2 / 1,2}\right)} \lambda_{2}<1,\right. \\
\left.\lambda_{2}<\mathcal{P}\left(\mathcal{D}_{2 / 1,2}\right)\right\} .
\end{gathered}
$$

Similarly, the stability region $\mathcal{R}_{2}$ obtained from the second dominant system, i.e., when the second queue transmits dummy packets is as follows:

$$
\begin{gathered}
\mathcal{R}_{2}=\left\{\left(\lambda_{1}, \lambda_{2}\right): \frac{\lambda_{2}}{\mathcal{P}\left(\mathcal{D}_{2 / 2}\right)}+\frac{\mathcal{P}\left(\mathcal{D}_{2 / 2}\right)-\mathcal{P}\left(\mathcal{D}_{2 / 1,2}\right)}{\mathcal{P}\left(\mathcal{D}_{2 / 2}\right) \mathcal{P}\left(\mathcal{D}_{1 / 1,2}^{s}\right)} \lambda_{1}<1,\right. \\
\left.\lambda_{1}<\mathcal{P}\left(\mathcal{D}_{1 / 1,2}^{s}\right)\right\} .
\end{gathered}
$$

The stability region of the system is obtained by taking union of $\mathcal{R}_{1}$ and $\mathcal{R}_{2}$, i.e., $\mathcal{R}=\mathcal{R}_{1} \cup \mathcal{R}_{2}$, where $\mathcal{R}_{1}$ and $\mathcal{R}_{2}$ correspond to the stability regions of dominant systems in (4) and (5), respectively. The stability region above is expressed as a function of the success probabilities without secrecy constraint, i.e., $\mathcal{P}\left(\mathcal{D}_{i / \mathcal{T}}\right)$, and with secrecy constraint, i.e., $\mathcal{P}\left(\mathcal{D}_{i / i, \mathcal{T}}^{s}\right)$. The stability region determined using (4) and (5) is general and no specific decoding scheme needs to be assumed at the receivers. Note that the queue stability conditions are necessary and sufficient for the stability of the original system as obtained in [16], and the proof is based on [2].

\section{Stability Region AnAlysis With DifFERENT DECODING SCHEMES AT THE RECEIVERS}

In this section, the success probabilities with and without secrecy constraints are obtained with different decoding schemes at the receivers to determine the stability region. In a multiuser environment, users can have different decoding capabilities due to different hardware, and this in turn can affect the overall system performance. To study the impact of different decoding capabilities of the receiver, secrecy constraint and self-interference on the stability region, the following cases are considered.
1) Both users have limited decoding capability and cannot perform successive decoding. In this case, both users decode their intended packets with treating interference as noise, i.e., they treat other user's packet as noise while decoding its own packet. $^{2}$

2) The receiver with full-duplex capability, i.e., $D_{1}$, can perform successive decoding while the other receiver $D_{2}$ treats interference as noise. In successive decoding, the receiver first tries to decode the packet of the unintended user, and then decodes its own packet after canceling the effect of the other user's packet.

3) $D_{1}$ cannot perform successive decoding and decodes its intended packet by treating interference as noise while the second receiver $D_{2}$ can perform successive decoding. The second and third cases are of interest for the scenarios, where one of the receivers has limited decoding capability and the other receiver can perform successive decoding. ${ }^{3}$

For the third case, it is assumed that if receiver 2 fails to decode the packet of first user with successive decoding, then it attempts to decode the packet of the other user by treating interference as noise. Recall that the packet intended for receiver 1 needs to be kept secret from receiver 2 . Receiver 2 can decode the packet intended to receiver 1 either with successive decoding or treating interference as noise. Hence, receiver 2 has additional decoding capability compared to receiver 1 which can decode its intended packet only with treating interference as noise. In the following section, the success probabilities for different decoding schemes are derived. Using the expressions for the success probabilities in (4) and (5), the stability region is obtained for different cases as discussed above. The feasibility conditions are also derived for the success probability under different decoding capability at the receiver. These conditions are useful for numerical evaluation of the stability region for different cases discussed in Section V.

\section{A. Both Receivers Treat Interference as Noise}

In this case, it is assumed that both receivers have limited decoding capability and cannot perform successive decoding. Hence, receiver 1 has to treat the received packet intended for receiver 2 and the residual jamming signal after selfinterference cancelation as noise to decode its intended packet. Similarly, receiver 2 treats both packets intended for receiver 1 and the jamming signal sent by receiver 1 as noise while decoding its intended packet. In the following, the success probabilities for both receivers are obtained with and without secrecy constraint for different status of the queues. We denote the threshold for decoding a packet at the receiver sent from the $i^{\text {th }}$ queue as $\gamma_{i}(i=1,2)$, the distance between the transmitter and the receiver $i$ as $d_{i}(i=1,2)$, the distance between the receivers as $d_{3}$, and the path loss exponent as $\alpha$.

\footnotetext{
${ }^{2}$ From practical perspective, treating interference as noise is an attractive choice for decoding in many real world applications due to its low complexity and robustness to channel uncertainty.

${ }^{3}$ This is also relevant when one of the receivers is far from the transmitter compared to the other receiver and is in general true for the broadcast channel.
} 
1) When $Q_{1}=0$ and $Q_{2} \neq 0$ : In this case, the second queue at the transmitter sends a packet for receiver 2 as $Q_{1}=$ 0 . Since there is no secrecy constraint for the packets intended to receiver 2 at queue 2 , the receiver 2 can decode its intended packet if the following event is true

$$
\mathcal{D}_{2 / 2}=\left\{P_{2}\left|h_{2}\right|^{2} d_{2}^{-\alpha} \geq \gamma_{2}\right\} \text {. }
$$

Note that receiver 1 does not send a jamming signal in this case since receiver 1 can know in advance that it is not going to receive packets from the first queue through exchange of control signals with transmitter. The success probability is then given by

$$
\begin{aligned}
\mathcal{P}\left(\mathcal{D}_{2 / 2}\right) & =1-\mathcal{P}\left\{P_{2}\left|h_{2}\right|^{2} d_{2}^{-\alpha}<\gamma_{2}\right\} \\
& =1-\int_{0}^{\infty} F_{\left|h_{2}\right|^{2}}\left(\frac{\gamma_{2}}{P_{2} d_{2}^{-\alpha}}\right) f_{\left|h_{2}\right|^{2}}(x) d x \\
& =\exp \left(-\frac{\gamma_{2} d_{2}^{\alpha}}{P_{2}}\right)
\end{aligned}
$$

where $F_{X}(x)$ is the $\mathrm{CDF}$ of the random variable $X$ and $f_{\left|h_{2}\right|^{2}}(x)=\exp (-x)$.

2) When $Q_{1} \neq 0$ and $Q_{2}=0$ : In this case, queue 2 is empty, so receiver 2 does not have a packet to decode from queue 2. However, receiver 2 tries to decode the packet intended to receiver 1 . Hence, there is an additional secrecy constraint compared to the previous case. The packet of queue 1 sent by transmitter should be decoded at receiver 1 while it should be kept confidential from receiver 2 , and this is presented by the following event

$$
\mathcal{D}_{1 / 1}^{s}=\left\{\frac{P_{1}\left|h_{1}\right|^{2} d_{1}^{-\alpha}}{1+P_{J} g^{2}} \geq \gamma_{1}, \frac{P_{1}\left|h_{2}\right|^{2} d_{2}^{-\alpha}}{1+P_{J}\left|h_{12}\right|^{2} d_{3}^{-\alpha}} \leq \gamma_{1}\right\} .
$$

Note that in [6], the secrecy of the message is based on an information theoretic approach. In this work, we consider finite packet length and thus, the secrecy criteria is defined in terms of SINR as in [37]-[42]. The SINR threshold $\gamma_{i}$ is determined by many factors/parameters of a communication system such as application, data rate, signal processing applied at encoder and decoder, and error correction coding [37]. In general, the secrecy capacity, outage probability or ergodic secrecy rate have been used as a metric for communication secrecy. However, these metrics are non-trivial to analyze when the block length is finite [43]. To overcome this problem, one can use the bit error rate (BER) over message bits as a metric for security of a communication system. The information theoretic results from finite block length coding can also be used to evaluate these metrics [44], [45]. One can look into packet error rate (PER), which can be a useful metric for cross layer (PHY/MAC) security design. The PER at a receiver is defined as the number of erroneously received packets to the total number of transmitted packets. For the packets sent from the first queue, a higher PER is desirable at receiver 2 from the security point of view and a lower PER is required at receiver 1 for reliable decoding of the packet. The packet error rate will be a function of the SINR threshold, channel condition, decoding capability at the receiver, and error correction coding. The objective here will be to ensure that receiver 2 operates above a certain PER level and receiver 1 operates at a relatively low PER value [27].

Since $h_{1}, h_{2}$ and $h_{12}$ are independent of each other, the success probability for receiver 1 is given by

$$
\begin{gathered}
\mathcal{P}\left(\mathcal{D}_{1 / 1}^{s}\right) \\
=\mathcal{P}\left\{\frac{P_{1}\left|h_{1}\right|^{2} d_{1}^{-\alpha}}{1+P_{J} g^{2}} \geq \gamma_{1}\right\} \mathcal{P}\left\{\frac{P_{1}\left|h_{2}\right|^{2} d_{2}^{-\alpha}}{1+P_{J}\left|h_{12}\right|^{2} d_{3}^{-\alpha}} \leq \gamma_{1}\right\}, \\
=\exp \left(-\frac{\gamma_{1} d_{1}^{\alpha}}{P_{1}}\left(1+P_{J} g^{2}\right)\right)\left\{1-\exp \left(-\frac{\gamma_{1} d_{2}^{\alpha}}{P_{1}}\right)\right. \\
\left.\times\left[1+\gamma_{1} \frac{P_{J}}{P_{1}}\left(\frac{d_{2}}{d_{3}}\right)^{\alpha}\right]^{-1}\right\} .
\end{gathered}
$$

3) When $Q_{1} \neq 0$ and $Q_{2} \neq 0$ : In this case, both queues have packets to transmit. The packet sent from the first queue should be decoded at receiver 1 and at the same time be confidential to receiver 2 , which is presented by the following event

$$
\begin{aligned}
\mathcal{D}_{1 / 1,2}^{s}=\{ & \frac{P_{1}\left|h_{1}\right|^{2} d_{1}^{-\alpha}}{1+P_{2}\left|h_{1}\right|^{2} d_{1}^{-\alpha}+P_{J} g^{2}} \geq \gamma_{1}, \\
& \left.\frac{P_{1}\left|h_{2}\right|^{2} d_{2}^{-\alpha}}{1+P_{2}\left|h_{2}\right|^{2} d_{2}^{-\alpha}+P_{J}\left|h_{12}\right|^{2} d_{3}^{-\alpha}}<\gamma_{1}\right\} .
\end{aligned}
$$

The success probability in this case becomes

$$
\begin{aligned}
& \mathcal{P}\left(\mathcal{D}_{1 / 1,2}^{s}\right) \\
& =\mathcal{P}\left\{\frac{P_{1}\left|h_{1}\right|^{2} d_{1}^{-\alpha}}{1+P_{2}\left|h_{1}\right|^{2} d_{1}^{-\alpha}+P_{J} g^{2}} \geq \gamma_{1},\right. \\
& \left.\frac{P_{1}\left|h_{2}\right|^{2} d_{2}^{-\alpha}}{1+P_{2}\left|h_{2}\right|^{2} d_{2}^{-\alpha}+P_{J}\left|h_{12}\right|^{2} d_{3}^{-\alpha}}<\gamma_{1}\right\}, \\
& =\mathcal{P}\left\{\frac{\left(P_{1}-\gamma_{1} P_{2}\right)\left|h_{1}\right|^{2} d_{1}^{-\alpha}}{1+P_{J} g^{2}} \geq \gamma_{1}\right\} \times \\
& \mathcal{P}\left\{\frac{\left(P_{1}-\gamma_{1} P_{2}\right)\left|h_{2}\right|^{2} d_{2}^{-\alpha}}{1+P_{J}\left|h_{12}\right|^{2} d_{3}^{-\alpha}}<\gamma_{1}\right\}, \\
& =\exp \left(-\frac{\gamma_{1} d_{1}^{\alpha}}{P_{1}-\gamma_{1} P_{2}}\left(1+P_{J} g^{2}\right)\right)\{1- \\
& \left.\exp \left(-\frac{\gamma_{1} d_{2}^{\alpha}}{P_{1}-\gamma_{1} P_{2}}\right)\left[1+\gamma_{1} \frac{P_{J}}{P_{1}-\gamma_{1} P_{2}}\left(\frac{d_{2}}{d_{3}}\right)^{\alpha}\right]^{-1}\right\} \text {. }
\end{aligned}
$$

From (11), we can see that the event $\mathcal{D}_{1 / 1,2}^{s}$ can occur with a non-zero probability if

$$
\frac{P_{1}}{P_{2}}>\gamma_{1}
$$

Receiver 2 can decode the packet sent by the second queue when the following event is true.

$$
\mathcal{D}_{2 / 1,2}=\left\{\frac{P_{2}\left|h_{2}\right|^{2} d_{2}^{-\alpha}}{1+P_{1}\left|h_{2}\right|^{2} d_{2}^{-\alpha}+P_{J}\left|h_{12}\right|^{2} d_{3}^{-\alpha}} \geq \gamma_{2}\right\} .
$$

The success probability in this case is

$$
\begin{aligned}
& \mathcal{P}\left(\mathcal{D}_{2 / 1,2}\right) \\
& =\exp \left(-\frac{\gamma_{2} d_{2}^{\alpha}}{P_{2}-\gamma_{2} P_{1}}\right)\left[1+\gamma_{2} \frac{P_{J}}{P_{2}-\gamma_{2} P_{1}}\left(\frac{d_{2}}{d_{3}}\right)^{\alpha}\right]^{-1} .
\end{aligned}
$$


The event $\mathcal{D}_{2 / 1,2}$ can occur with nonzero probability if the following condition is satisfied

$$
\frac{P_{2}}{P_{1}}>\gamma_{2}
$$

Hence, both events $\mathcal{D}_{1 / 1,2}^{s}$ and $\mathcal{D}_{2 / 1,2}$ can occur, provided the conditions in (12) and (15) are satisfied.

Using (9), (11) and (14), the stability region in (4) becomes (16). Using (7), (11) and (14), the stability region in (5) becomes (17). Finally, the stability region of the system with secrecy constraint is obtained by taking union of $\mathcal{R}_{1}$ and $\mathcal{R}_{2}$ in (16) and (17), respectively. Note that (12) and (15) are useful for the numerical evaluation of the stability region of the system.

Now, we consider the cases when the receivers have different decoding capabilities. Note that, when $\left(Q_{1} \neq 0, Q_{2}=0\right)$ and $\left(Q_{1}=0, Q_{2} \neq 0\right)$, the probability of successful decoding for receivers 1 and 2 are given by (9) and (7), respectively. However, the expressions of probability of successful decoding are different for all cases, when both queues have packet to send. Hence, in the following, we have considered the case when both queues have packet to send $\left(Q_{1} \neq 0\right.$ and $\left.Q_{2} \neq 0\right)$.

\section{B. Receiver 1 Performs Successive Decoding and Receiver 2 Treats Interference as Noise}

In this subsection, we consider the case, where receiver 1 performs successive decoding [46], i.e., it first decodes the packet intended to receiver 2 , and cancels its effect from the output. Then, receiver 1 tries to decode its intended packet. The second receiver cannot perform successive decoding, and hence treats other user's packet as noise while decoding its intended packet. The expression for success probability in case of $\left(Q_{1} \neq 0, Q_{2} \neq 0\right)$ is obtained as follows.

$$
\begin{gathered}
\mathcal{D}_{1 / 2,1}^{s}=\left\{\frac{P_{2}\left|h_{1}\right|^{2} d_{1}^{-\alpha}}{1+P_{1}\left|h_{1}\right|^{2} d_{1}^{-\alpha}+g^{2} P_{J}} \geq \gamma_{2}, \frac{P_{1}\left|h_{1}\right|^{2} d_{1}^{-\alpha}}{1+g^{2} P_{J}} \geq \gamma_{1}\right. \\
\left.\frac{P_{1}\left|h_{2}\right|^{2} d_{2}^{-\alpha}}{1+P_{2}\left|h_{2}\right|^{2} d_{2}^{-\alpha}+\left|h_{12}\right|^{2} d_{3}^{-\alpha} P_{J}}<\gamma_{1}\right\} .
\end{gathered}
$$

The success probability of receiver 1 is then given by

$$
\begin{aligned}
\mathcal{P}\left(\mathcal{D}_{1 / 2,1}^{s}\right) & \mathcal{P}\left(\left|h_{1}\right|^{2} \geq \max \left\{\frac{\gamma_{2} d_{1}^{\alpha}}{P_{2}-\gamma_{2} P_{1}}, \frac{\gamma_{1} d_{1}^{\alpha}}{P_{1}}\right\}\right) \times \\
& \mathcal{P}\left(\left|h_{2}\right|^{2} \leq \frac{\gamma_{1} d_{2}^{\alpha}}{P_{1}-\gamma_{1} P_{2}}\right), \\
= & \exp \left(-\max \left\{\frac{\gamma_{2}\left(1+g^{2} P_{J}\right) d_{1}^{\alpha}}{P_{2}-\gamma_{2} P_{1}}, \frac{\gamma_{1}\left(1+g^{2} P_{J}\right) d_{1}^{\alpha}}{P_{1}}\right\}\right) \times \\
& {\left[1-\exp \left(-\frac{\gamma_{1} d_{2}^{\alpha}}{P_{1}-\gamma_{1} P_{2}}\right)\left\{1+\gamma_{1} \frac{P_{J}}{P_{1}-\gamma_{1} P_{2}}\left(\frac{d_{2}}{d_{3}}\right)^{\alpha}\right\}^{-1}\right] . }
\end{aligned}
$$

As receiver 2 decodes its intended packet by treating other user's packet as noise, the success probability for receiver 2 is given by (14).

Using the success probabilities obtained for different cases in (4) and (5), the stability region for successive decoding scheme can be obtained.
C. Receiver 1 Treats Interference as Noise and Receiver 2 Performs Successive Decoding

In this section, we consider the case when receiver 1 cannot perform successive decoding. Hence, it decodes its own packet by treating interference as noise. It is assumed that receiver 2 can perform successive decoding. In this case, receiver 2 decodes the packet of the first queue and then, tries to decode its intended packet, i.e., the packet sent from the second queue. If receiver 2 fails in successive decoding, then it attempts to decode its intended packet by treating interference as noise. The jamming signal sent by receiver 1 creates additional noise at receiver 2 and hence, it can assist receiver 1 to achieve higher secrecy throughput. However, the jamming signal transmitted by receiver 1 creates self-interference at receiver 1 and the strength of the interference depends on the degree of self-interference cancelation. Receiver 1, while decoding its own packet, treats both packets intended for receiver 2 and the residual self-interference signal as noise. In the following, the success probability for both receivers are obtained when both queues have packet to send.

1) When $Q_{1} \neq 0$ and $Q_{2} \neq 0$ : In this case we assume that receiver 2 can perform successive decoding. For the event $\mathcal{D}_{1 / 1,2}^{s}$ to be true, receiver 1 should be able to decode its own packet and receiver 2 should not be able to decode the packet intended to receiver 1 either by treating interference as noise or successive decoding. In the case of successive decoding, receiver 2 tries to decode its intended packet first and then tries to cancel its effect to decode the packet sent from queue 1. However, in both cases, the jamming signal sent from receiver 1 remains as noise at receiver 2 .

The probability that receiver 1 decodes its intended packet with the secrecy constraint is determined as

$$
\begin{aligned}
& \mathcal{P}\left(\mathcal{D}_{1 / 1,2}^{s}\right)= \mathcal{P}\left(\mathcal{D}_{1 / 1,2}^{s, 2 \mathrm{SD}}\right)+\mathcal{P}\left(\mathcal{D}_{1 / 1,2}^{s, 2 \mathrm{TIN}}\right)- \\
& \mathcal{P}\left(\mathcal{D}_{1 / 1,2}^{s, 2 \mathrm{SD}} \cap \mathcal{D}_{1 / 1,2}^{s, 2 \mathrm{TIN}}\right),
\end{aligned}
$$

where $\mathcal{D}_{1 / 1,2}^{s, 2 \mathrm{SD}}$ denotes the event that receiver 1 receives secretly its information if receiver 2 can perform successive decoding (SD), i.e., receiver 2 decodes its intended packet first, then tries to decode the packet intended to receiver 1 , and $\mathcal{D}_{1 / 1,2}^{s, 2}$ denotes the event that receiver 1 can decode its information secretly if receiver 2 performs treating interference as noise (TIN).

The probability of the event $\mathcal{D}_{1 / 1,2}^{s, 2 \mathrm{SD}}$ is calculated as follows

$$
\begin{aligned}
\mathcal{P}\left(\mathcal{D}_{1 / 1,2}^{s, 2 \mathrm{SD}}\right) & =\mathcal{P}\left(A_{1} \cap A_{2} \cap A_{3}\right), \\
& =\mathcal{P}\left(A_{1} \mid A_{2} \cap A_{3}\right) \mathcal{P}\left(A_{2} \cap A_{3}\right), \\
& =\mathcal{P}\left(A_{1}\right) \mathcal{P}\left(A_{2} \cap A_{3}\right),
\end{aligned}
$$

where the events $A_{1}, A_{2}$ and $A_{3}$ are defined as

$$
\begin{aligned}
A_{1} & \triangleq\left\{\frac{P_{1}\left|h_{1}\right|^{2} d_{1}^{-\alpha}}{1+P_{2}\left|h_{1}\right|^{2} d_{1}^{-\alpha}+P_{J} g^{2}} \geq \gamma_{1}\right\} \\
A_{2} & \triangleq\left\{\frac{P_{2}\left|h_{2}\right|^{2} d_{2}^{-\alpha}}{1+P_{1}\left|h_{2}\right|^{2} d_{2}^{-\alpha}+P_{J}\left|h_{12}\right|^{2} d_{3}^{-\alpha}} \geq \gamma_{2}\right\}, \\
\text { and } A_{3} & \triangleq\left\{\frac{P_{1}\left|h_{2}\right|^{2} d_{2}^{-\alpha}}{1+P_{J}\left|h_{12}\right|^{2} d_{3}^{-\alpha}}<\gamma_{1}\right\}
\end{aligned}
$$




$$
\begin{aligned}
& \mathcal{R}_{1}=\left\{\begin{array}{c}
\left(\lambda_{1}, \lambda_{2}\right): \frac{1-\exp \left(-\gamma_{1} d_{1}^{\alpha}\left(1+g^{2} P_{J}\right)\left(\frac{1}{P_{1}-\gamma_{1} P_{2}}-\frac{1}{P_{1}}\right)\right) \frac{1-\exp \left(-\frac{\gamma_{1} d_{2}^{\alpha}}{P_{1}}\right)\left[1+\gamma_{1} \frac{P_{J}}{P_{1}}\left(\frac{d_{2}}{d_{3}}\right)^{\alpha}\right]^{-1}}{1-\exp \left(-\frac{\gamma_{1} d_{2}^{\alpha}}{P_{1}-\gamma_{1} P_{2}}\right)\left[1+\gamma_{1} \frac{P_{J}}{P_{1}-\gamma_{1} P_{2}}\left(\frac{d_{2}}{d_{3}}\right)^{\alpha}\right]^{-1}}}{\exp \left(-\frac{\gamma_{2} d_{2}^{\alpha}}{P_{2}-\gamma_{2} P_{1}}\right)\left[1+\gamma_{2} \frac{P_{J}}{P_{2}-\gamma_{2} P_{1}}\left(\frac{d_{2}}{d_{3}}\right)^{\alpha}\right]^{-1}} \lambda_{2}
\end{array}\right. \\
& \left.+\frac{\lambda_{1}}{\exp \left(-\frac{\gamma_{1} d_{1}^{\alpha}}{P_{1}}\left(1+g^{2} P_{J}\right)\right)\left\{1-\exp \left(-\frac{\gamma_{1} d_{2}^{\alpha}}{P_{1}}\right)\left[1+\gamma_{1} \frac{P_{J}}{P_{1}}\left(\frac{d_{2}}{d_{3}}\right)^{\alpha}\right]^{-1}\right\}}<1, \lambda_{2}<\frac{\exp \left(-\frac{\gamma_{2} d_{2}^{\alpha}}{P_{2}-\gamma_{2} P_{1}}\right)}{1+\gamma_{2} \frac{P_{J}}{P_{2}-\gamma_{2} P_{1}}\left(\frac{d_{2}}{d_{3}}\right)^{\alpha}}\right\} . \\
& \mathcal{R}_{2}=\left\{\left(\lambda_{1}, \lambda_{2}\right): \frac{1-\exp \left(-\left(\frac{1}{P_{2}-\gamma_{2} P_{1}}-\frac{1}{P_{2}}\right) \gamma_{2} d_{2}^{\alpha}\right)\left(\frac{1+\gamma_{2} \frac{P_{J}}{P_{2}-\gamma_{2} P_{1}}\left(\frac{d_{2}}{d_{3}}\right)^{\alpha}}{1+\gamma_{2} \frac{P_{J}}{P_{2}}\left(\frac{d_{2}}{d_{3}}\right)^{\alpha}}\right)^{-1}}{\exp \left(-\frac{\gamma_{1} d_{1}^{\alpha}}{P_{1}-\gamma_{1} P_{2}}\left(1+g^{2} P_{J}\right)\right)\left\{1-\exp \left(-\frac{\gamma_{1} d_{2}^{\alpha}}{P_{2}-\gamma_{2} P_{1}}\right)\left[1+\gamma_{1} \frac{P_{J}}{P_{1}-\gamma_{1} P_{2}}\left(\frac{d_{2}}{d_{3}}\right)^{\alpha}\right]^{-1} \lambda_{1}\right.} \lambda_{1}\right. \\
& +\frac{\lambda_{2}}{\exp \left(-\frac{\gamma_{2} d_{2}^{\alpha}}{P_{2}}\right)\left[1+\gamma_{2} \frac{P_{J}}{P_{2}}\left(\frac{d_{2}}{d_{3}}\right)^{\alpha}\right]^{-1}}<1, \\
& \left.\lambda_{1} \leq \exp \left(-\frac{\gamma_{1} d_{1}^{\alpha}}{P_{1}-\gamma_{1} P_{2}}\left(1+g^{2} P_{J}\right)\right)\left\{1-\exp \left(-\frac{\gamma_{1} d_{2}^{\alpha}}{P_{2}-\gamma_{2} P_{1}}\right)\left[1+\gamma_{1} \frac{P_{J}}{P_{1}-\gamma_{1} P_{2}}\left(\frac{d_{2}}{d_{3}}\right)^{\alpha}\right]^{-1}\right\}\right\} .
\end{aligned}
$$

Note that in the above, $A_{1}$ is independent of $A_{2}$ and $A_{3}$. Now, we consider the evaluation of the following term in (21)

$$
\begin{aligned}
\mathcal{P}\left(A_{1}\right) & =\mathcal{P}\left\{\frac{P_{1}\left|h_{1}\right|^{2} d_{1}^{-\alpha}}{1+P_{2}\left|h_{1}\right|^{2} d_{1}^{-\alpha}+P_{J} g^{2}} \geq \gamma_{1}\right\}, \\
& =\exp \left(-\frac{\gamma_{1} d_{1}^{\alpha}}{P_{1}-\gamma_{1} P_{2}}\left(1+P_{J} g^{2}\right)\right) .
\end{aligned}
$$

Consider the evaluation of the second term in (21)

$$
\begin{gathered}
\mathcal{P}\left(A_{2} \cap A_{3}\right) \\
=\mathcal{P}\left\{\frac{P_{2}\left|h_{2}\right|^{2} d_{2}^{-\alpha}}{1+P_{1}\left|h_{2}\right|^{2} d_{2}^{-\alpha}+P_{J}\left|h_{12}\right|^{2} d_{3}^{-\alpha}} \geq \gamma_{2},\right. \\
\left.\frac{P_{1}\left|h_{2}\right|^{2} d_{2}^{-\alpha}}{1+P_{J}\left|h_{12}\right|^{2} d_{3}^{-\alpha}}<\gamma_{1}\right\} .
\end{gathered}
$$

For the event $\left\{A_{2} \cap A_{3}\right\}$ to be feasible, the following condition is required to be satisfied

$$
\frac{P_{1}}{P_{2}}<\frac{\gamma_{1}}{\left(1+\gamma_{1}\right) \gamma_{2}} .
$$

On further simplification, $\mathcal{P}\left(A_{2} \cap A_{3}\right)$ becomes

$$
\begin{aligned}
\mathcal{P}\left(A_{2} \cap A_{3}\right) & \\
= & \mathcal{P}\left(\frac{\gamma_{2}\left(1+P_{J}\left|h_{12}\right|^{2} d_{3}^{-\alpha}\right)}{\left(P_{2}-\gamma_{2} P_{1}\right) d_{2}^{-\alpha}} \leq\left|h_{2}\right|^{2} \leq\right. \\
& \left.\quad \frac{\gamma_{1}\left(1+P_{J}\left|h_{12}\right|^{2} d_{3}^{-\alpha}\right)}{P_{1} d_{2}^{-\alpha}}\right), \\
= & \int_{0}^{\infty} \mathcal{P}\left(\frac{\gamma_{2}\left(1+P_{J}\left|h_{12}\right|^{2} d_{3}^{-\alpha}\right)}{\left(P_{2}-\gamma_{2} P_{1}\right) d_{2}^{-\alpha}} \leq\left|h_{2}\right|^{2}\right. \\
& \left.\left.\quad \frac{\gamma_{1}\left(1+P_{J}\left|h_{12}\right|^{2} d_{3}^{-\alpha}\right)}{P_{1} d_{2}^{-\alpha}}|| h_{12}\right|^{2}=x\right) f_{\left|h_{12}\right|^{2}}(x) \mathrm{d} x \\
= & \frac{\exp \left(-\frac{\gamma_{2} d_{2}^{\alpha}}{P_{2}-\gamma_{2} P_{1}}\right)}{1+\frac{\gamma_{2} P_{J}}{P_{2}-\gamma_{2} P_{1}}\left(\frac{d_{2}}{d_{3}}\right)^{\alpha}}-\frac{\exp \left(-\frac{\gamma_{1} d_{2}^{\alpha}}{P_{1}}\right)}{1+\gamma_{1} \frac{P_{J}}{P_{1}}\left(\frac{d_{2}}{d_{3}}\right)^{\alpha}},
\end{aligned}
$$

where for the above integral to exist the following condition must be satisfied

$$
\left(\frac{d_{2}}{d_{3}}\right)^{\alpha}>\frac{\gamma_{2} P_{1}-P_{2}}{\gamma_{2} P_{J}} .
$$

Using (23) and (26), (21) becomes

$$
\begin{gathered}
\mathcal{P}\left(\mathcal{D}_{1 / 1,2}^{s, 2 \mathrm{SD}}\right)=\exp \left(-\frac{\gamma_{1} d_{1}^{\alpha}}{P_{1}-\gamma_{1} P_{2}}\left(1+P_{J} g^{2}\right)\right) \times \\
{\left[\frac{\exp \left(\frac{-\gamma_{2} d_{2}^{\alpha}}{P_{2}-\gamma_{2} P_{1}}\right)}{1+\frac{\gamma_{2} P_{J}}{P_{2}-\gamma_{2} P_{1}}\left(\frac{d_{2}}{d_{3}}\right)^{\alpha}}-\frac{\exp \left(\frac{-\gamma_{1} d_{2}^{\alpha}}{P_{1}}\right)}{1+\gamma_{1} \frac{P_{J}}{P_{1}}\left(\frac{d_{2}}{d_{3}}\right)^{\alpha}}\right] .}
\end{gathered}
$$


Now, consider the evaluation of $\mathcal{P}\left(\mathcal{D}_{1 / 1,2}^{s, 2 \text { TIN }}\right)$ in (20)

$$
\begin{aligned}
\mathcal{P}\left(\mathcal{D}_{1 / 1,2}^{s, 2 \text { TIN }}\right) & =\mathcal{P}\left(A_{1}\right) \mathcal{P}\left(A_{4} \cap A_{5}\right), \\
= & \exp \left(-\frac{\gamma_{1} d_{1}^{\alpha}}{P_{1}-\gamma_{1} P_{2}}\left(1+P_{J} g^{2}\right)\right) \mathcal{P}\left(A_{4} \cap A_{5}\right),
\end{aligned}
$$

where the events $A_{4}$ and $A_{5}$ are defined as

$$
\begin{aligned}
& A_{4}=\left\{\frac{P_{2}\left|h_{2}\right|^{2} d_{2}^{-\alpha}}{1+P_{1}\left|h_{2}\right|^{2} d_{2}^{-\alpha}+P_{J}\left|h_{12}\right|^{2} d_{3}^{-\alpha}}<\gamma_{2}\right\}, \text { and } \\
& A_{5}=\left\{\frac{P_{1}\left|h_{2}\right|^{2} d_{2}^{-\alpha}}{1+P_{2}\left|h_{2}\right|^{2} d_{2}^{-\alpha}+P_{J}\left|h_{12}\right|^{2} d_{3}^{-\alpha}}<\gamma_{1}\right\} .
\end{aligned}
$$

Consider the following

$$
\begin{aligned}
& \mathcal{P}\left(A_{4} \cap A_{5}\right) \\
& =\mathcal{P}\left\{\frac{P_{2}\left|h_{2}\right|^{2} d_{2}^{-\alpha}}{1+P_{1}\left|h_{2}\right|^{2} d_{2}^{-\alpha}+P_{J}\left|h_{12}\right|^{2} d_{3}^{-\alpha}}<\gamma_{2},\right. \\
& =\mathcal{P}\left\{\frac{P_{1}\left|h_{2}\right|^{2} d_{2}^{-\alpha}}{1+P_{2}\left|h_{2}\right|^{2} d_{2}^{-\alpha}+P_{J}\left|h_{12}\right|^{2} d_{3}^{-\alpha}}<\gamma_{1}\right\}, \\
& \left.=1-\exp \left(-\frac{\left|h_{2}\right|^{2}}{\max \left\{\frac{P_{2}}{\gamma_{2}}-P_{1}, \frac{P_{1}}{\gamma_{1}}-P_{2}\right\}}\right) \times \frac{\left.d_{2}^{\alpha}\right|^{2} d_{3}^{-\alpha}}{\max \left\{\frac{P_{2}}{\gamma_{2}}-P_{1}, \frac{P_{1}}{\gamma_{1}}-P_{2}\right\}}\right\}, \\
& \left\{1+\frac{d_{2}^{\alpha}}{\max \left\{\frac{P_{2}}{\gamma_{2}}-P_{1}, \frac{P_{1}}{\gamma_{1}}-P_{2}\right\}} P_{J} d_{3}^{-\alpha}\right\}
\end{aligned}
$$

Using (31), (29) becomes

$$
\begin{aligned}
& \mathcal{P}\left(\mathcal{D}_{1 / 1,2}^{s, 2 \text { TIN }}\right) \\
& =\exp \left(-\frac{\gamma_{1} d_{1}^{\alpha}}{P_{1}-\gamma_{1} P_{2}}\left(1+P_{J} g^{2}\right)\right) \times \\
& \left(1-\exp \left(-\frac{d_{2}^{\alpha}}{\max \left\{\frac{P_{2}}{\gamma_{2}}-P_{1}, \frac{P_{1}}{\gamma_{1}}-P_{2}\right\}}\right) \times\right. \\
& \left.\left\{1+\frac{d_{2}^{\alpha}}{\max \left\{\frac{P_{2}}{\gamma_{2}}-P_{1}, \frac{P_{1}}{\gamma_{1}}-P_{2}\right\}} P_{J} d_{3}^{-\alpha}\right\}^{-1}\right) .
\end{aligned}
$$

It can be noticed that as the events $A_{2}$ and $A_{4}$ are mutually exclusive of each other, we have

$$
\mathcal{P}\left(\mathcal{D}_{1 / 1,2}^{s, 2 \mathrm{SD}} \cap \mathcal{D}_{1 / 1,2}^{s, 2 \text { TIN }}\right)=0
$$

Using (28), (32), and (33), (20) becomes

$$
\begin{aligned}
& \mathcal{P}\left(\mathcal{D}_{1 / 1,2}^{s}\right) \\
& =\exp \left(-\frac{\gamma_{1} d_{1}^{\alpha}}{P_{1}-\gamma_{1} P_{2}}\left(1+P_{J} g^{2}\right)\right)\left[\frac{\exp \left(\frac{-\gamma_{2} d_{2}^{\alpha}}{P_{2}-\gamma_{2} P_{1}}\right)}{1+\frac{\gamma_{2} P_{J}}{P_{2}-\gamma_{2} P_{1}}\left(\frac{d_{2}}{d_{3}}\right)^{\alpha}}-\right. \\
& \frac{\exp \left(\frac{-\gamma_{1} d_{2}^{\alpha}}{P_{1}}\right)}{1+\gamma_{1} \frac{P_{J}}{P_{1}}\left(\frac{d_{2}}{d_{3}}\right)^{\alpha}}+\exp \left(-\frac{\gamma_{1} d_{1}^{\alpha}}{P_{1}-\gamma_{1} P_{2}}\left(1+P_{J} g^{2}\right)\right) \times \\
& \left(1-\exp \left(-\frac{d_{2}^{\alpha}}{\max \left\{\frac{P_{2}}{\gamma_{2}}-P_{1}, \frac{P_{1}}{\gamma_{1}}-P_{2}\right\}}\right)^{-1} \times\right. \\
& \left\{\begin{array}{c}
\left.1+\frac{d_{2}^{\alpha}}{\max \left\{\frac{P_{2}}{\gamma_{2}}-P_{1}, \frac{P_{1}}{\gamma_{1}}-P_{2}\right\}} P_{J} d_{3}^{-\alpha}\right\}
\end{array} .\right.
\end{aligned}
$$

In the following, the success probabilities for receiver 2 is obtained. In this case, receiver 2 first attempts to decode its intended packet by successive decoding. If it fails in successive decoding, it decodes by treating interference as noise.

$$
\begin{aligned}
\mathcal{P}\left(\mathcal{D}_{2 \mid 1,2}\right) & =\mathcal{P}\left(\mathcal{D}_{2 \mid 1,2}^{2 \mathrm{TIN}} \cup \mathcal{D}_{2 \mid 1,2}^{2 \mathrm{SD}}\right), \\
& =\mathcal{P}\left(\mathcal{D}_{2 \mid 1,2}^{2 \mathrm{TIN}}\right)+\mathcal{P}\left(\mathcal{D}_{2 \mid 1,2}^{2 \mathrm{SD}}\right)-\mathcal{P}\left(\mathcal{D}_{2 \mid 1,2}^{2 \mathrm{TIN}} \cap \mathcal{D}_{2 \mid 1,2}^{2 \mathrm{SD}}\right),
\end{aligned}
$$

where $\mathcal{D}_{2 \mid 1,2}^{2 \text { TIN }}$ denotes the event that receiver 2 is able to decode its intended packet with treating interference as noise and $\mathcal{D}_{2 \mid 1,2}^{2 \mathrm{SD}}$ denotes the event that receiver 2 is able to decode its intended packet with successive decoding, i.e., receiver 2 decodes the packet intended to receiver 1 , then attempts to decode its own packet. To calculate $\mathcal{P}\left(\mathcal{D}_{2 \mid 1,2}\right)$, consider the following events

$$
\begin{aligned}
& B_{1} \triangleq\left\{\frac{P_{2}\left|h_{2}\right|^{2} d_{2}^{-\alpha}}{1+P_{1}\left|h_{2}\right|^{2} d_{2}^{-\alpha}+P_{J}\left|h_{12}\right|^{2} d_{3}^{-\alpha}} \geq \gamma_{2}\right\}, \\
& B_{2} \triangleq\left\{\frac{P_{1}\left|h_{2}\right|^{2} d_{2}^{-\alpha}}{1+P_{2}\left|h_{2}\right|^{2} d_{2}^{-\alpha}+P_{J}\left|h_{12}\right|^{2} d_{3}^{-\alpha}} \geq \gamma_{1}\right\}, \\
& \text { and } B_{3} \triangleq\left\{\frac{P_{2}\left|h_{2}\right|^{2} d_{2}^{-\alpha}}{\left.1+P_{J}\left|h_{12}\right|^{2} d_{3}^{-\alpha} \geq \gamma_{2}\right\}}\right.
\end{aligned}
$$

Now, consider the following

$$
\begin{aligned}
& \mathcal{P}\left(\mathcal{D}_{2 \mid 1,2}^{2 \mathrm{TIN}}\right)=\mathcal{P}\left(B_{1}\right) \\
& =\exp \left(-\frac{\gamma_{2} d_{2}^{\alpha}}{P_{2}-\gamma_{2} P_{1}}\right)\left[1+\gamma_{2} \frac{P_{J}}{P_{2}-\gamma_{2} P_{1}}\left(\frac{d_{2}}{d_{3}}\right)^{\alpha}\right]^{-1}
\end{aligned}
$$

The equation above is obtained using the results derived in Section III-A (See (13) and (14)).

Consider the calculation of probability of the event $\mathcal{D}_{2 \mid 1,2}^{2 \mathrm{SD}}=$ $B_{2} \cap B_{3} \cap B_{1}^{c}$, where $B_{1}^{c}$ denotes complement of the event $B_{1}$ defined in (36). Since the events $\mathcal{D}_{2 \mid 1,2}^{2 \text { TIN }}$ and $\mathcal{D}_{2 \mid 1,2}^{2 \mathrm{SD}}$ are mutually 
exclusive, we have

$$
\begin{aligned}
& \mathcal{P}\left(\mathcal{D}_{2 \mid 1,2}^{2 \mathrm{SD}}\right)=\mathcal{P}\left(B_{2} \cap B_{3} \cap B_{1}^{c}\right), \\
& =\mathcal{P}\left(\frac{P_{1}\left|h_{2}\right|^{2} d_{2}^{-\alpha}}{1+P_{2}\left|h_{2}\right|^{2} d_{2}^{-\alpha}+P_{J}\left|h_{12}\right|^{2} d_{3}^{-\alpha}} \geq \gamma_{1},\right. \\
& \frac{P_{2}\left|h_{2}\right|^{2} d_{2}^{-\alpha}}{1+P_{J}\left|h_{12}\right|^{2} d_{3}^{-\alpha}} \geq \gamma_{2}, \\
& \left.\frac{P_{2}\left|h_{2}\right|^{2} d_{2}^{-\alpha}}{1+P_{1}\left|h_{2}\right|^{2} d_{2}^{-\alpha}+P_{J}\left|h_{12}\right|^{2} d_{3}^{-\alpha}}<\gamma_{2}\right), \\
& =\mathcal{P}\left(\gamma_{2}^{\prime} \leq \frac{\left|h_{2}\right|^{2}}{1+P_{J}\left|h_{12}\right|^{2} d_{3}^{-\alpha}} \leq \gamma_{2}^{\prime \prime}\right),
\end{aligned}
$$

where $\gamma_{2}^{\prime} \triangleq \min \left\{\frac{\gamma_{1} d_{2}^{\alpha}}{P_{1}-\gamma_{1} P_{2}}, \frac{\gamma_{2} d_{2}^{\alpha}}{P_{2}}\right\}$ and $\gamma_{2}^{\prime \prime}=\frac{\gamma_{2} d_{2}^{\alpha}}{P_{2}-\gamma_{2} P_{1}}$. For this probability to exist, following condition is required to be satisfied

$$
\frac{\gamma_{1}}{P_{1}-\gamma_{1} P_{2}}<\frac{\gamma_{2}}{P_{2}-\gamma_{2} P_{1}} .
$$

When (39) is satisfied, (38) becomes

$$
\begin{aligned}
& \mathcal{P}\left(\mathcal{D}_{2 \mid 1,2}^{2 \mathrm{SD}}\right) \\
& =\mathcal{P}\left(\gamma_{2}^{\prime}\left(1+P_{J}\left|h_{12}\right|^{2} d_{3}^{-\alpha}\right) \leq\left|h_{2}\right|^{2} \leq\right. \\
& \left.\gamma_{2}^{\prime \prime}\left(1+P_{J}\left|h_{12}\right|^{2} d_{3}^{-\alpha}\right)\right), \\
& =\int_{0}^{\infty} \mathcal{P}\left(\gamma_{2}^{\prime}\left(1+P_{J}\left|h_{12}\right|^{2} d_{3}^{-\alpha}\right) \leq\left|h_{2}\right|^{2}\right. \\
& \left.\quad<\left.\gamma_{2}^{\prime \prime}\left(1+P_{J}\left|h_{12}\right|^{2} d_{3}^{-\alpha}\right)|| h_{12}\right|^{2}=x\right) f_{\left|h_{12}\right|^{2}}(x) \mathrm{d} x \\
& =\frac{\exp \left(-\gamma_{2}^{\prime}\right)}{1+\gamma_{2}^{\prime} P_{J} d_{3}^{-\alpha}}-\frac{\exp \left(-\gamma_{2}^{\prime \prime}\right)}{1+\gamma_{2}^{\prime \prime} P_{J} d_{3}^{-\alpha}} .
\end{aligned}
$$

Using (37) and (40) and noticing that $\mathcal{P}\left(\mathcal{D}_{2 \mid 1,2}^{2 \mathrm{TIN}} \cap \mathcal{D}_{2 \mid 1,2}^{2 \mathrm{SD}}\right)=$ 0 , (35) becomes

$$
\begin{gathered}
\mathcal{P}\left(\mathcal{D}_{2 \mid 1,2}\right) \\
=\exp \left(-\frac{\gamma_{2} d_{2}^{\alpha}}{P_{2}-\gamma_{2} P_{1}}\right)\left[1+\gamma_{2} \frac{P_{J}}{P_{2}-\gamma_{2} P_{1}}\left(\frac{d_{2}}{d_{3}}\right)^{\alpha}\right]^{-1} \\
\quad+\frac{\exp \left(-\gamma_{2}^{\prime}\right)}{1+\gamma_{2}^{\prime} P_{J} d_{3}^{-\alpha}}-\frac{\exp \left(-\gamma_{2}^{\prime \prime}\right)}{1+\gamma_{2}^{\prime \prime} P_{J} d_{3}^{-\alpha}}
\end{gathered}
$$

Replacing the success probabilities obtained for different cases in (4) and (5), the stability region can be determined.

\section{Closure of the Stability Region}

In Section III, the stability regions were obtained using success probability for fixed powers at the transmitter and the jammer (receiver 1). It was also found that when the selfinterference cancelation is not efficient, the gain obtained by jamming can be diminished. A way to overcome this problem is to allocate different transmit powers for the queues at the source and at jammer, which can make the stability region larger. In this subsection, we explore how the stability region can be improved by power allocation.
1) Closure of the stability region over all possible power allocation at the Source: Previously, we presented the stability region for given power allocation. In this subsection, we present the stability region over all possible power allocations at the transmitter/source, i.e., $P_{1} \in[0, P], P_{2} \in[0, P]$, for a given total power constraint, i.e., $P_{1}+P_{2}=P$. However, the jamming power $\left(P_{J}\right)$ is kept fixed. The closure of the stability region over all possible power allocations is defined as

$$
\begin{aligned}
\mathcal{C}\left(P_{J}\right) \triangleq & \left(\bigcup_{\left(P_{1}, P_{2}\right) \in[0, P]^{2}, P_{1}+P_{2}=P} \mathcal{C}_{1}\left(P_{1}, P_{2}, P_{J}\right)\right) \\
& \bigcup\left(\bigcup_{\left(P_{1}, P_{2}\right) \in[0, P]^{2}, P_{1}+P_{2}=P} \mathcal{C}_{2}\left(P_{1}, P_{2}, P_{J}\right)\right),
\end{aligned}
$$

where $\mathcal{C}_{i}\left(P_{1}, P_{2}, P_{J}\right) \triangleq \mathcal{R}_{i}$ for $i=1,2$. In (42), we take the union of the regions over all possible power allocations for each queue corresponding to the closure of the stability region.

2) Closure of the stability region over all possible jamming powers at Receiver 1: In this case, the power at the jammer is varied and the power allocation at the transmitter is kept fixed. The closure of the stability region in this case is defined as

$$
\begin{aligned}
& \mathcal{C}\left(P_{1}, P_{2}\right) \triangleq\left(\bigcup_{P_{J} \in\left[0, P_{J}^{\max }\right]} \mathcal{C}_{1}\left(P_{1}, P_{2}, P_{J}\right)\right) \\
& \bigcup\left(\bigcup_{P_{J} \in\left[0, P_{J}^{\max }\right]} \mathcal{C}_{2}\left(P_{1}, P_{2}, P_{J}\right)\right),
\end{aligned}
$$

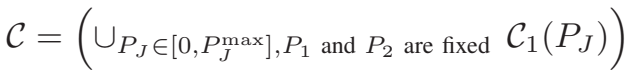

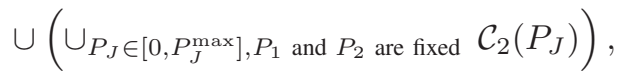

where $\mathcal{C}_{i}\left(P_{1}, P_{2}, P_{J}\right) \triangleq \mathcal{R}_{i}$ for $i=1,2$.

Note that in both cases, $\mathcal{R}_{i}$ can correspond to the stability region obtained for different decoding schemes in Sections III-A-III-C. The impact of power control on the stability region is illustrated in Figs. 6-11.

\section{E. Remarks}

1) The stability region for the $\mathrm{BC}$ with secrecy constraint when receiver 1 does not have full-duplex capability can be obtained as a special case by setting $P_{J}=0$ for different decoding schemes.

2) In the third case (Section III-C), if receiver 2 can only perform successive decoding (i.e., it cannot treat interference as noise if it fails in successive decoding), then the success probabilities $\mathcal{P}\left(\mathcal{D}_{1 / 1,2}^{s}\right)$ and $\mathcal{P}\left(\mathcal{D}_{2 \mid 1,2}\right)$ are obtained by setting $\mathcal{P}\left(\mathcal{D}_{1 / 1,2}^{s, 2 \text { TIN }}\right)=0$ and $\mathcal{P}\left(\mathcal{D}_{2 \mid 1,2}^{2 \mathrm{TIN}}\right)=0$ in (20) and (35), respectively.

3) The results derived in this section are also useful for other communication scenarios such as the cases with different kind of data arrivals and congestion levels at the queues. We can also apply the results for the optimal 
design of the system. For example, the jamming power of receiver 1 can be optimized to enhance the system throughput as we present in Section IV.

4) By following similar steps as in the third scenario (Section III-C), it is possible to obtain the stability region for the scenario (Section III-B), where if receiver 1 fails in successive decoding can still perform treating interference as noise. It may enlarge the stability region of the 2-user $\mathrm{BC}$ compared to the case where receiver 1 can only perform successive decoding.

5) The exact characterization of the stability region of networks with bursty sources is known to be a difficult problem due to the interaction among the queues (e.g., each node/queue transmits, and thus, interferes with others when its queue is non-empty). The characterization of the stability region with $N$-queues $(N>2)$ for basic models like multiple access channel has remained as an open problem even without secrecy constraints [19]. Hence, extension of the results developed in this work to more than 2-receivers is non-trivial and an interesting avenue for research.

6) Using the result in [46, Theorem 5.3], it is not difficult to show that both receivers cannot perform successive decoding simultaneously since it results in infeasible power allocation even for the BC without the secrecy constraint as described below. The capacity of two user Gaussian BC without secrecy constraint is given by

$$
\begin{aligned}
& R_{1} \leq \log \left(1+\left|h_{1}\right|^{2} P_{1}\right), \\
& R_{2} \leq \log \left(1+\frac{\left|h_{2}\right|^{2} P_{2}}{1+\left|h_{2}\right|^{2} P_{1}}\right) .
\end{aligned}
$$

From the above result one can say that both receivers cannot perform successive decoding. If both users can perform successive decoding, then both users should be able to achieve a rate of $R_{i}=\log \left(1+\left|h_{i}\right|^{2} P_{i}\right), i=1,2$. For this to be possible the following should be true for receiver 2

$$
\begin{aligned}
& \quad \log \left(1+\left|h_{2}\right|^{2} P_{2}\right)<\log \left(1+\frac{\left|h_{1}\right|^{2} P_{2}}{1+\left|h_{2}\right|^{2} P_{1}}\right), \\
& \text { or } P_{2}<\frac{\left|h_{2}\right|^{2}-\left|h_{1}\right|^{2}}{\left|h_{2}\right|^{2}\left|h_{1}\right|^{2}} .
\end{aligned}
$$

The above condition ensures that the message intended to receiver 2 is also decodable at receiver 1 . For receiver 1 , the following condition is required to be satisfied

$$
\begin{aligned}
& \quad \log \left(1+\left|h_{1}\right|^{2} P_{1}\right)<\log \left(1+\frac{\left|h_{2}\right|^{2} P_{1}}{1+\left|h_{2}\right|^{2} P_{2}}\right), \\
& \text { or } P_{1}<\frac{\left|h_{1}\right|^{2}-\left|h_{2}\right|^{2}}{\left|h_{2}\right|^{2}\left|h_{1}\right|^{2}} .
\end{aligned}
$$

From (47) and (48), it can be concluded for successive decoding at both receivers, it results in infeasible power allocations. The above result is obtained using information theoretic result and it is expected that for finite backlogged users, the probability of successful decoding will be low and can result in poor performance. This is also observed for the broadcast channel without secrecy constraint [16].

7) Recently, there has been an increased interest in caching with and without secrecy constraint in networks [47][49], which prefetches some of the popular contents in distributed caches for low latency. The framework of this paper can also be used as a building block to explore the effect of caching on the stability of the system with secrecy constraints for characterizing the performance of secure caching with bursty traffic.

\section{Optimal JAMMing POWER Design For MAXIMIZING SATURATED THROUGHPUT OF RECEIVER 2}

In this section, we assume that queue 2 always has packets to send while packets arrive at queue 1 at a rate $\lambda_{1}$. As queue 2 never empties, we have $\mathcal{P}\left(Q_{2}>0\right)=1$. The objective here is to maximize the saturated throughput for receiver 2 by choosing the jamming power such that a minimum service rate is guaranteed for queue 1 . The jamming signal transmitted by receiver 1 may increase the service rate for queue 1 and also can decrease the saturated throughput for receiver 2 . It is important to note that the self-interference caused at receiver 1 may also decrease the service rate seen at the first queue if the self-interference cancelation technique is not efficient. Hence, it is non-trivial to solve the optimization problem. The average service rate for the first queue is

$$
\mu_{1}=\mathcal{P}\left(\mathcal{D}_{1 / 1,2}^{s}\right)
$$

The average service rate for the second queue is

$$
\mu_{2}=\mathcal{P}\left(Q_{1}>0\right) \mathcal{P}\left(\mathcal{D}_{2 / 1,2}\right)+\mathcal{P}\left(Q_{1}=0\right) \mathcal{P}\left(\mathcal{D}_{2 / 2}\right),
$$

where $\mathcal{P}\left(Q_{1}>0\right)=\frac{\lambda_{1}}{\mathcal{P}\left(\mathcal{D}_{1 / 1,2}^{s}\right)}$. Since, the saturated throughput for queue 2 is determined by the service rate, it is given by

$$
\mu_{2}=\mathcal{P}\left(\mathcal{D}_{2 / 2}\right)-\frac{\mathcal{P}\left(\mathcal{D}_{2 / 2}\right)-\mathcal{P}\left(\mathcal{D}_{2 / 1,2}\right)}{\mathcal{P}\left(\mathcal{D}_{1 / 1,2}^{s}\right)} \lambda_{1}
$$

Finally, the optimization problem can be stated as follows

$$
\begin{aligned}
& \max _{P_{J}} \mathcal{P}\left(\mathcal{D}_{2 / 2}\right)-\frac{\mathcal{P}\left(\mathcal{D}_{2 / 2}\right)-\mathcal{P}\left(\mathcal{D}_{2 / 1,2}\right)}{\mathcal{P}\left(\mathcal{D}_{1 / 1,2}^{s}\right)} \lambda_{1}, \\
& \text { s.t. } \quad 0 \leq P_{J} \leq P_{J}^{\max }, \text { and } \mu_{1} \geq \mu_{t h} .
\end{aligned}
$$

where $P_{J}^{\max }$ is the maximum power budget at receiver 1 . In (53), the first condition ensures that the power constraint at receiver 2 is satisfied and the second condition ensures that the service rate for queue 1 is at least $\mu_{t h}$. The case of saturated and non-saturated queues can occur in wireless sensor networks where one of the queues receive sensitive information from some specific sensors. Hence, its arrival rate is expected to be random in nature as the sensors may not always have sensitive information to send. The other queue receives general information from many sources and thus, it results in congestion at the second queue. The sensitive information at queue 1 needs to be sent to receiver 1 under a minimum service rate constraint without violating secrecy 


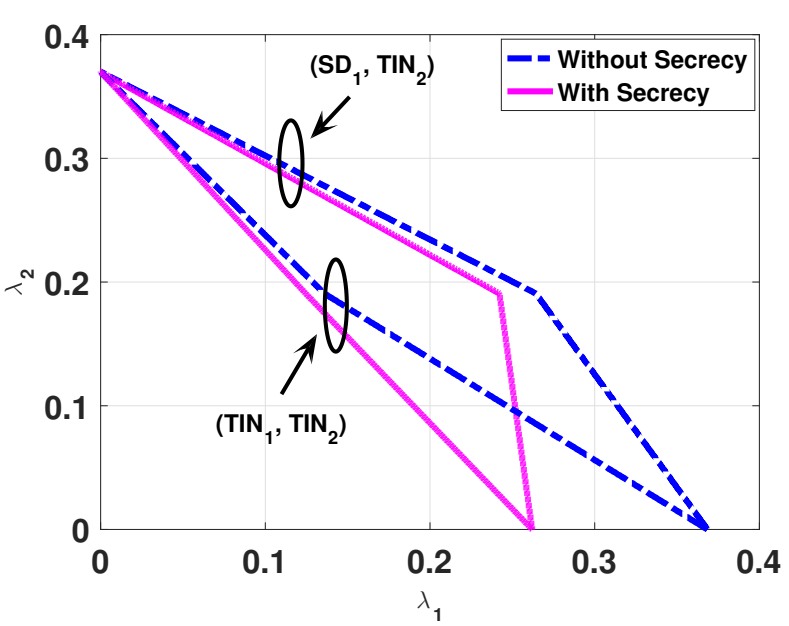

Fig. 2. The stability region where $P_{1}=P_{2}=100, P_{J}=0, \gamma_{1}=0.5$, $\gamma_{2}=0.4, d=10, d_{1}=d, d_{2}=1.1 d$, and $\alpha=2.3$.

constraint. The problem considered in this section optimizes the jamming power to maximize the throughput of the user without a secrecy constraint under the constraint that the user with secrecy constraint will be guaranteed to achieve a desired service rate.

\section{NumERicAl RESUlts}

In this section, numerical results are presented first for the stability region for different decoding schemes at the receivers. Then, the closures of the stability regions are obtained for the different cases. Finally, numerical results are presented for the optimization problem of jamming power considered in Section IV.

\section{A. Stability Region Analysis with Different Decoding Schemes}

In this subsection, we present the stability region for different decoding schemes at receivers. In all figures, $\left(\mathrm{DC}_{1}, \mathrm{DC}_{2}\right)$ represents that the decoding scheme at receiver $i$ is $\mathrm{DC}_{i}$, which is either decoding by treating interference as noise (TIN) or successive decoding (SD).

1) No full-duplex capability at receiver 1: The stability regions are plotted in Fig. 2 when receiver 1 does not send any jamming signal to receiver 2 , i.e., $P_{J}=0$, and receiver 2 decodes by treating interference as noise (TIN) due to a decoding constraint. In this figure, different decoding capabilities for receiver 1 (i.e., SD and TIN) are considered for both cases with/without the secrecy constraint at receiver 1 . Note that the stability regions without the secrecy constraint are plotted based on [17] for both decoding schemes of receiver 1 . The stability regions with the secrecy constraints are plotted using the results derived in Sections III-A and III-B.

In Fig. 2, the transmit power for packet in the queues are assumed to be $P_{1}=P_{2}=100$. From Fig. 2, it can be noticed that due to the secrecy constraint for packets intended to receiver 1 , the stability region has reduced compared to the case when there is no secrecy constraint at receiver 1 . One can also notice that as the value of $\lambda_{1}$ decreases, the penalty on the stability region due to the secrecy constraint also decreases.

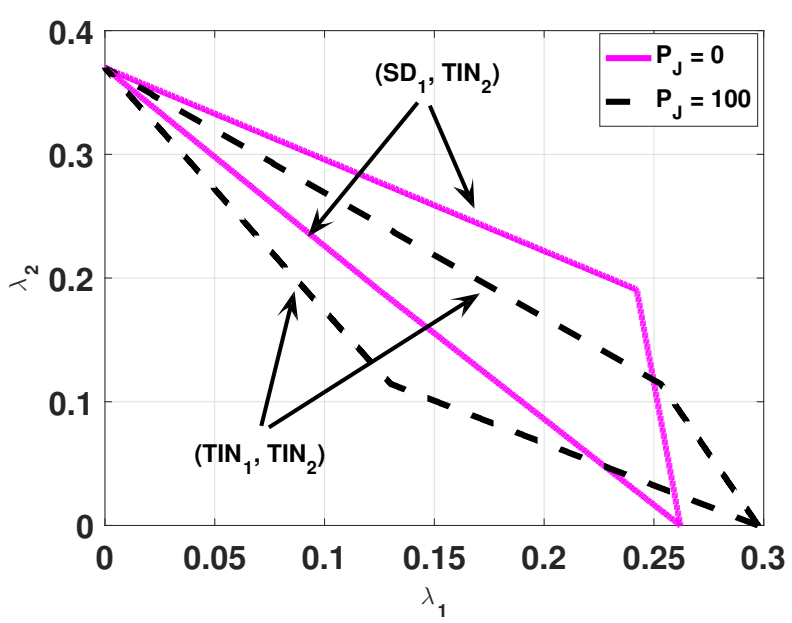

Fig. 3. The stability region with and without full-duplex capability at receiver 1: $P_{1}=100, P_{2}=100, \gamma_{1}=0.5, \gamma_{2}=0.4, d=10, d_{1}=d$, $d_{2}=d_{3}=1.1 d, g=10^{-3}$, and $\alpha=2.3$.

When there is no packet for receiver 1, there is no penalty on the throughput for receiver 2 as there is no secrecy constraint for the packet intended to receiver 2. It is interesting to note that the stability region with secrecy constraint for successive decoding can be larger than the stability region without secrecy constraint for the TIN case, for some values of $\lambda_{1}$ and $\lambda_{2}$.

2) With full-duplex capability at receiver 1: In this case, the stability region is depicted when receiver 1 sends a jamming signal to receiver 2 and it is assumed that receiver 2 cannot perform successive decoding. In Fig. 3, the stability regions are plotted using the results derived in Sections III-A (when both receivers treat interference as noise) and III-B (when receiver 1 performs successive decoding and receiver 2 treats interference as noise) for different values of jamming powers, when the coefficient of self-interference cancelation is $g=10^{-3}$ or $\beta \triangleq$ $-10 \log g^{2}=60 d B$. It can be noticed that with an increase in the power of the jamming signal, there is also an increase in the maximum achievable throughput for receiver 1 . However, it happens only in a small region for which the maximum achievable throughput for receiver 2 is small.

Next we consider the scenario when receiver 1 cannot perform successive decoding. Thus, it treats the packet of the second queue and the residual jamming signal as noise while decoding its intended packet. However, receiver 2 can perform successive decoding in this case. The SINR thresholds for decoding packets intended to receiver 1 and 2 are $\gamma_{1}=0.2$ and $\gamma_{2}=0.25$, respectively. In Fig. 4, the stability region obtained in Section III-C is plotted for $P_{1}=P_{2}=P_{J}=100$, and $\beta=60 \mathrm{~dB}$. From the plot, it can be noticed that it is possible to ensure nonzero stable throughput for queue 1, although receiver 2 has the capability to perform successive decoding. As the jamming power increases, receiver 1 can support larger arrival rates. However, the arrival rate for receiver 2 decreases with the increase in the jamming power as it causes additional interference at receiver 2 . 


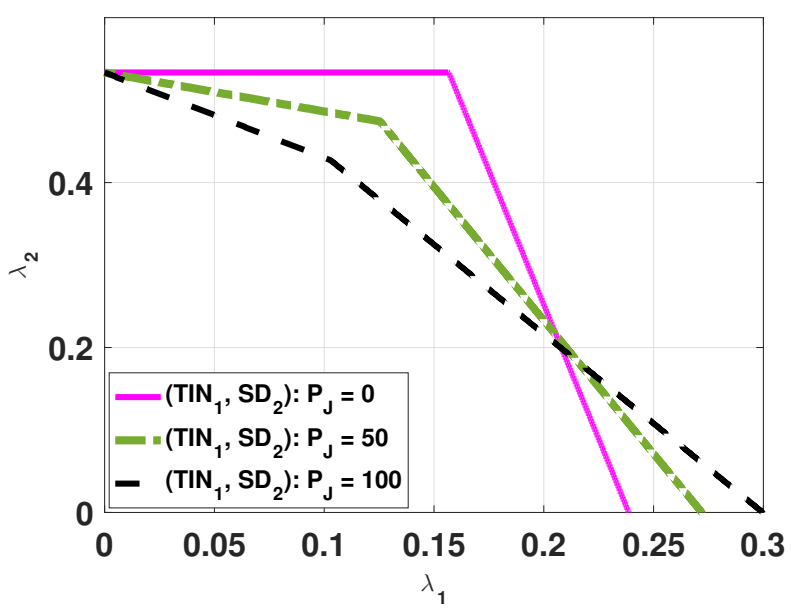

Fig. 4. The stability region when $R x-1$ treats interference as noise and Rx 2 can perform successive decoding: $P_{1}=P_{2}=100, \alpha=2.8, d=10$, $c=0.8, c^{\prime}=1.1, d_{1}=d, d_{2}=c d, d_{3}=c^{\prime} d, \gamma_{1}=0.2, \gamma_{2}=0.25$, and $\beta=60 d B$.

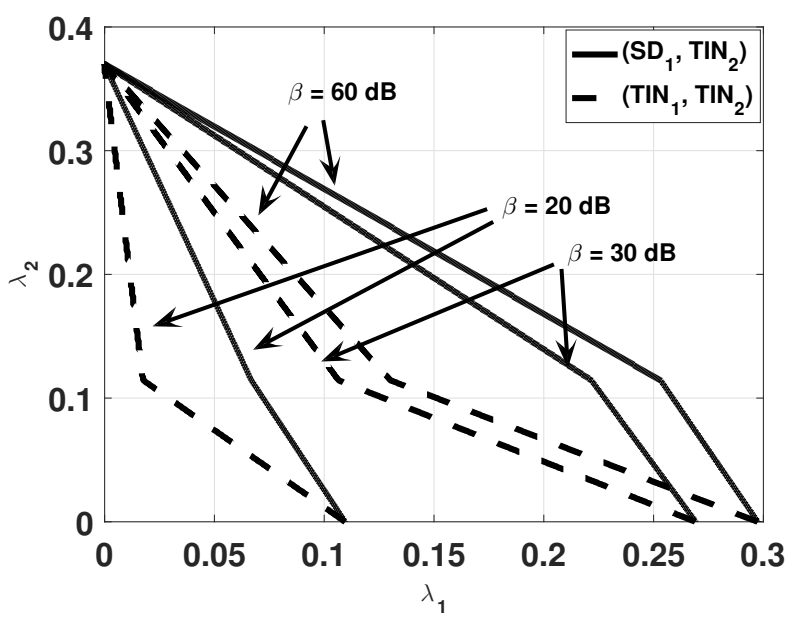

Fig. 5. The stability region for different levels of self-interference isolation: $P_{1}=P_{2}=100, P_{J}=100, \gamma_{1}=0.5, \gamma_{2}=0.4, d=10, d_{1}=d$, $d_{2}=d_{3}=1.1 d$, and $\alpha=2.3$.

\section{B. Impact of Imperfect Self-interference Cancelation on the Stability Region}

In this subsection, the effect of imperfect self-interference cancelation on the stability region is investigated. It is assumed that receiver 2 cannot perform successive decoding. In Fig. 5, the stability regions are plotted for different values of coefficient of self-interference cancelation when power of the jamming signal is kept fixed. The stability regions for $\left(\mathrm{TIN}_{1}, \mathrm{TIN}_{2}\right)$ and $\left(\mathrm{SD}_{1}, \mathrm{TIN}_{2}\right)$ are obtained using the results in Sections III-A and III-B, respectively. It can be noticed that as the value of $\beta$ increases, the stability region enlarges. Note that higher the value of $\beta$, the better is the self-interference cancelation technique at the full-duplex receiver. Hence, if the self-interference technique is not efficient, the benefits obtained in service rate due to the ability of receiver 1 sending jamming signal to receiver 2 can diminish for both decoding schemes.

The numerical results for the other case $\left(\mathrm{TIN}_{1}, \mathrm{SD}_{2}\right)$ can

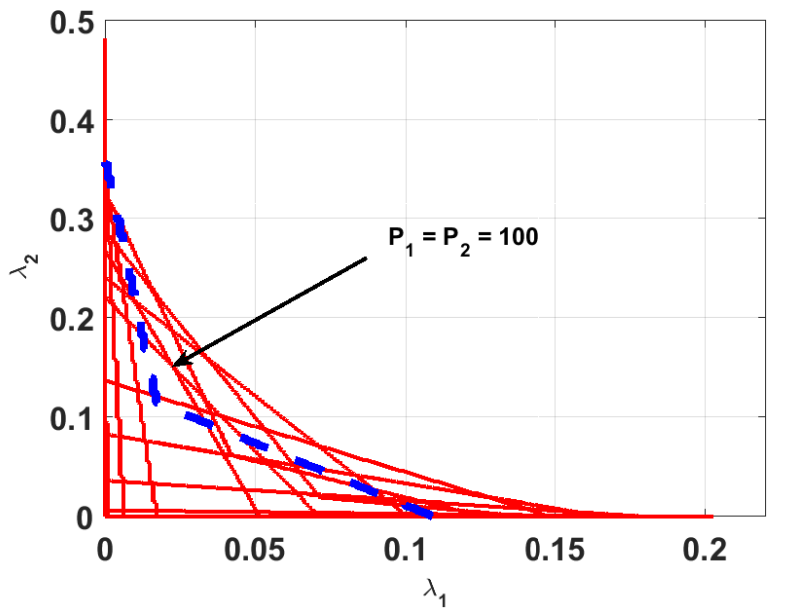

Fig. 6. Closure of the stability region when both receivers treat interference as noise and power allocations at both queues are varied: $P=200, P_{J}=100$, $\alpha=2.3, d=10, c=1.1, d_{1}=d, d_{2}=c d, d_{3}=c d, \gamma_{1}=0.5, \gamma_{2}=0.4$, and $\beta=20 d B$.

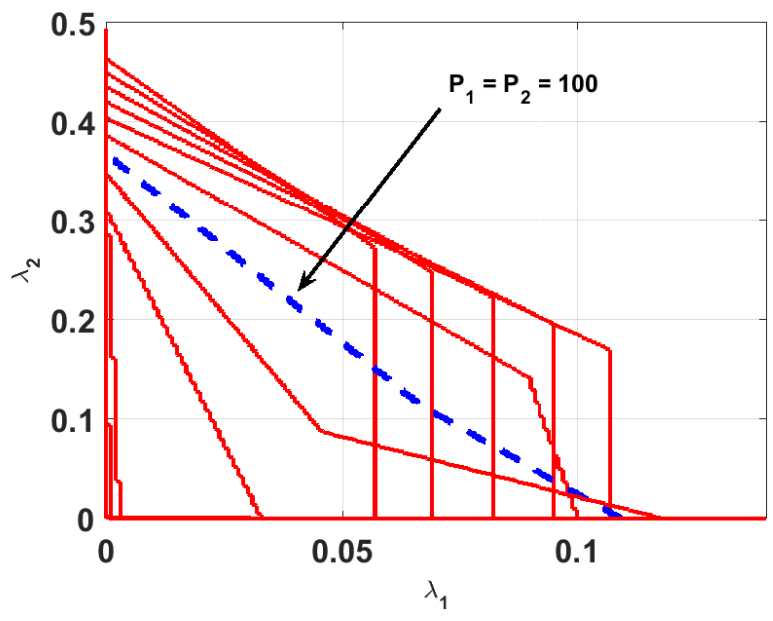

Fig. 7. Closure of the stability region when receiver 1 performs successive decoding while receiver 2 treats interference as noise and power allocations at both queues are varied: $P=200, P_{J}=100, \alpha=2.3, d=10, c=1.1$, $c^{\prime}=1.1, d_{1}=d, d_{2}=c d, d_{3}=c^{\prime} d, \gamma_{1}=0.5, \gamma_{2}=0.4$, and $\beta=20 d B$.

be obtained in a similar manner as obtained in case of $\left(\mathrm{SD}_{1}, \mathrm{TIN}_{2}\right)$. In this case, receiver 1 cannot perform successive decoding and hence, the packet of the other user will remain as noise at receiver 1 . In addition to that, the gain obtained in the service rate for receiver 1 due to the jamming signal sent by receiver 1 can diminish if the self-interference cancelation is not perfect.

Note that these results depict the stability region for one possible power allocation scheme. Each depicted stability region is the union of the two sub-regions described by (4) and (5) after replacing the appropriate expressions for the success probabilities depending on the decoding scheme. As it is union of two sub-regions, the stability regions in these plots are piecewise linear. 


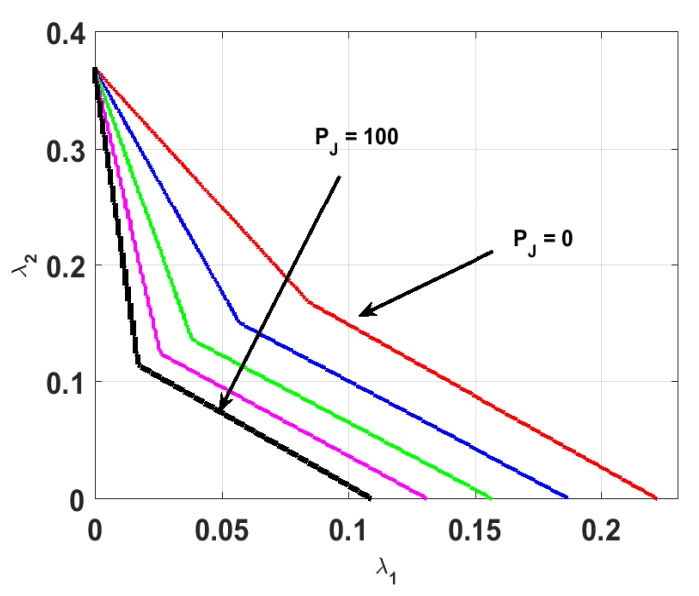

Fig. 8. Closure of the stability region when both receivers treat interference as noise and jamming power is varied $\left(0<P_{J} \leq 100\right): P_{1}=P_{2}=100$, $\alpha=2.3, d=10, c=1.1, d_{1}=d, d_{2}=c d, d_{3}=c d, \gamma_{1}=0.5, \gamma_{2}=0.4$, and $\beta=20 d B$.

\section{Closure of the stability region}

In the following, the closure of the stability region is plotted for different cases assuming that receiver 2 cannot perform successive decoding. ${ }^{4}$ First, consider the case when the transmitter has a total power constraint $P=200$ and the power allocations at both queues are varied under the following power constraint at the transmitter $P_{1}+P_{2}=P$. In this case, the jamming power at receiver 1 is kept fixed. We obtain the closure of the stability region under two types of decoding schemes: both receivers treat interference as noise (see Fig. 6) and receiver 1 performs successive decoding while receiver 2 decodes its intended packet by treating other user's packet as noise (see Fig. 7). In both cases, the jamming signal remains as noise at the receivers. From both plots, it can be noticed that it is possible to achieve a broader stability region with power control at the transmitter. However, power control is not very helpful to enlarge the stability region, when interference is treated as noise at both receivers.

Now, consider the second case, when the jamming power at receiver 1 is varied but the fixed power allocation is used at the transmitter for both queues. In this case, we also obtain the closure of the stability region under two types of decoding schemes: both receivers treat interference as noise (see Figs. 8-9) and receiver 1 performs successive decoding while receiver 2 decodes its intended packet by treating other user's packet as noise (see Figs. 10-11). Note that the degree of self-interference cancelation is $\beta=20 d B$ for Figs. 8 and 10 and $\beta=60 d B$ for Fig. 9 and 11. If the self-interference cancelation is poor, such as $\beta=20 \mathrm{~dB}$, we observe that as the jamming power increases the stability region shrinks since the legitimate user cannot cope with the increasing jamming power and eventually it makes its performance worse for the sake of secrecy. On the other hand, when the self-interference capability is better, i.e., when $\beta=60 \mathrm{~dB}$, we have a more

\footnotetext{
${ }^{4}$ One can also obtain the results for the case when receiver 2 performs successive decoding and receiver 1 treats interference as noise using the results in Section III-C.
}

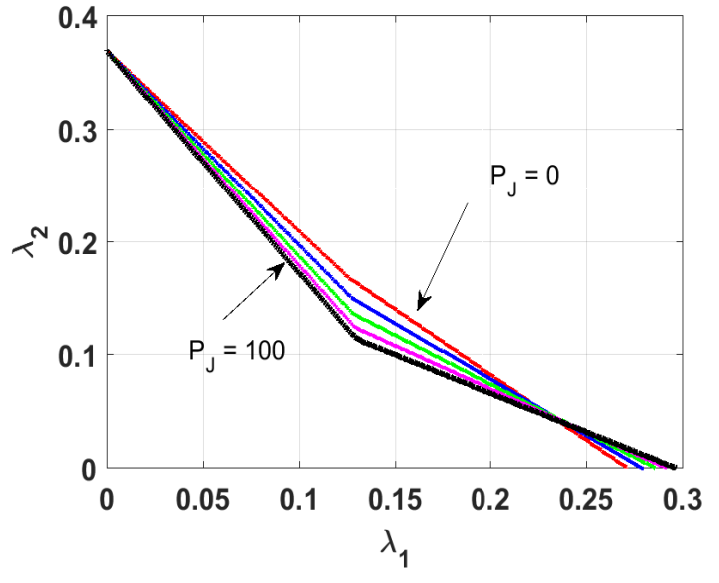

Fig. 9. Closure of the stability region when both receivers treat interference as noise and jamming power is varied $\left(0<P_{J} \leq 100\right): P_{1}=P_{2}=100$, $\alpha=2.3, d=10, c=1.1, d_{1}=d, d_{2}=c d, d_{3}=c d, \gamma_{1}=0.5, \gamma_{2}=0.4$, and $\beta=60 d B$.

interesting behavior regarding stability of the system. We observe that when we are on the high traffic regime for the legitimate user, and at the same time on the low traffic regime for the eavesdropper, the stable throughput performance is better. This is because the legitimate user can protect its packet better by canceling its self-interference since the eavesdropper does not have a lot of traffic and its queue is often empty. In particular, when the self-interference technique is not very efficient, the power control can be quite helpful to enlarge the stability region. Figs. 6-11 present the closure of the stability regions over all possible power allocations, and this can be seen as a network-level rate region of the system measured in packets/slot.

From Figs. 2, 3 and 5, it can be noticed that the stability regions can be convex or non-convex due to many factors such as decoding scheme at the receiver, jamming power and the degree of self-interference cancelation at receiver 1 . In Figs. 6 and 7, the stability regions are either convex or nonconvex depending on the power allocation at the queues. In Figs 8 and 9, it can be observed that the stability regions are non-convex for various values of $P_{J}$ when both users are treating interference as noise. When the stability region is not convex, the time sharing scheme can outperform the simultaneous transmission.

\section{Maximizing saturated throughput of receiver 2 under min- imum service rate for receiver 1}

In the following, we evaluate numerically the optimization problem in (52). It is assumed that the individual power allocation at both queues are $P_{1}=P_{2}=100$ and is fixed. The maximum power budget at receiver 1 is $P_{J}^{\max }=100$. For a desired service rate for queue 1, i.e., $\mu_{t h}$, we maximize the throughput $\mu_{2}$ for receiver 2 with respect to $P_{J}$, assuming that queue 2 is saturated. In Figs. 12 and 13, the saturated throughput and the jamming power are plotted versus $\mu_{t h}$, respectively. The success probabilities for different cases in (52) are evaluated using the results derived in Sec. III-A. From 


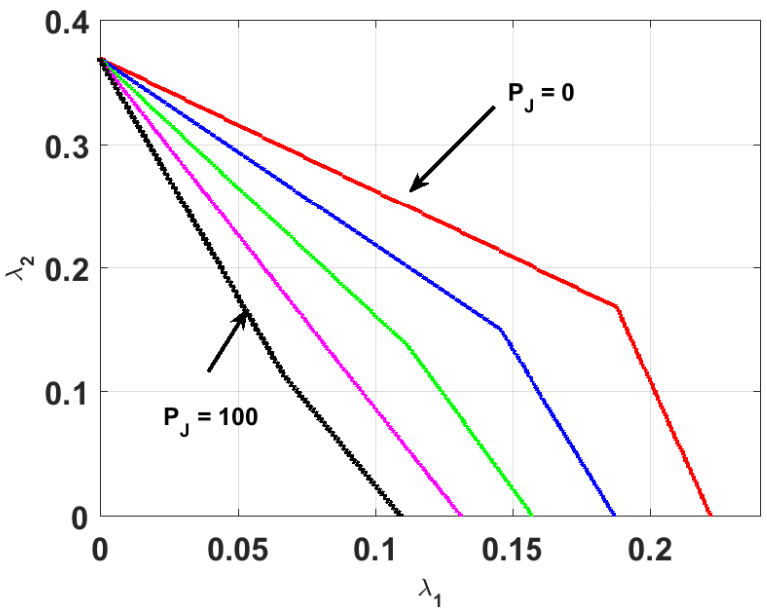

Fig. 10. Closure of the stability region when Rx 1 performs successive decoding, Rx 2 performs treating interference as noise and jamming power is varied $\left(0<P_{J} \leq 100\right): P=100, \alpha=2.3, d=10, c=1.1, c^{\prime}=1.1$, $d_{1}=d, d_{2}=c d, d_{3}=c^{\prime} d, \gamma_{1}=0.5, \gamma_{2}=0.4$, and $\beta=20 d B$.

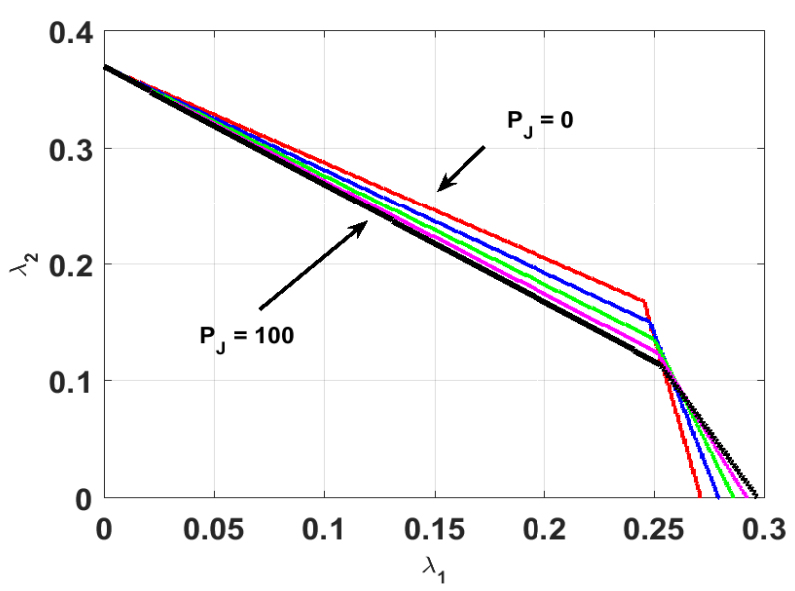

Fig. 11. Closure of the stability region when $\mathrm{Rx} 1$ performs successive decoding, Rx 2 performs treating interference as noise and jamming power is varied $\left(0<P_{J} \leq 100\right): P=100, \alpha=2.3, d=10, c=1.1, c^{\prime}=1.1$, $d_{1}=d, d_{2}=c d, d_{3}=c^{\prime} d, \gamma_{1}=0.5, \gamma_{2}=0.4$, and $\beta=60 d B$.

the plots, it can be noticed that when $\mu_{t h} \leq 0.125$, receiver 1 does not need to send a jamming signal to guarantee minimum service rate for queue 1 . However, when $\mu_{t h}>0.125$, receiver 1 needs to send a jamming signal of different power levels to guarantee minimum service rate for queue 1 as shown in Fig. 13. This leads to decrease in the saturated throughput for receiver 2 as $\mu_{t h}$ increases beyond 0.125 .

\section{CONCLUSION}

In this work, the stability region of the two-user broadcast channel is studied, where one of the receivers with secrecy constraint has full-duplex capability. The stability region of the $\mathrm{BC}$ is obtained for the general case. Then, the stability region is obtained for different decoding schemes at the receivers. The analysis takes account of the secrecy constraint at the receiver and impact of imperfect self-interference cancelation on the stability region simultaneously. When receiver 2 can

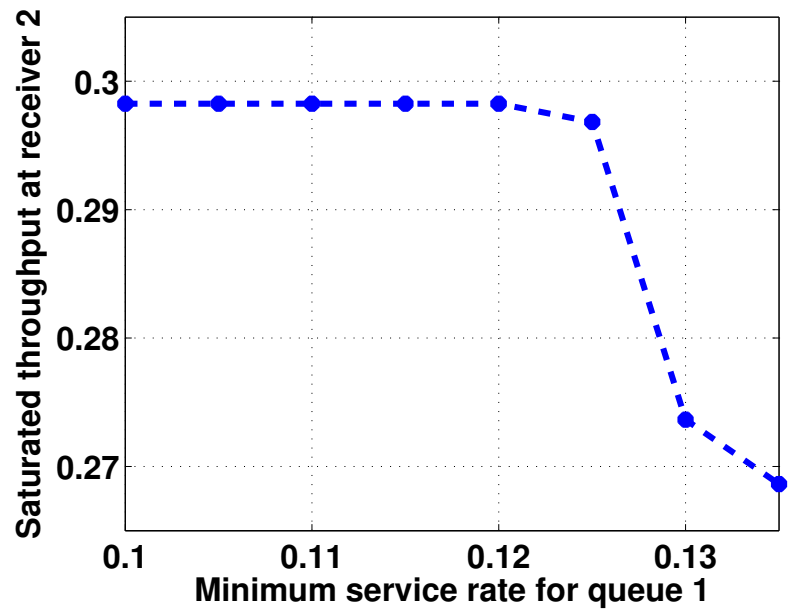

Fig. 12. Saturated throughput at receiver $2\left(\mu_{2}\right)$ vs Minimum service rate $\left(\mu_{t h}\right): P_{1}=P_{2}=100, P_{J}^{\max }=100, \alpha=2.2, d=10, c=1.1, d_{1}=d$, $d_{2}=c d, d_{3}=d, \gamma_{1}=0.5, \gamma_{2}=0.4, \lambda_{1}=0.05$, and $\beta=80 d B$.

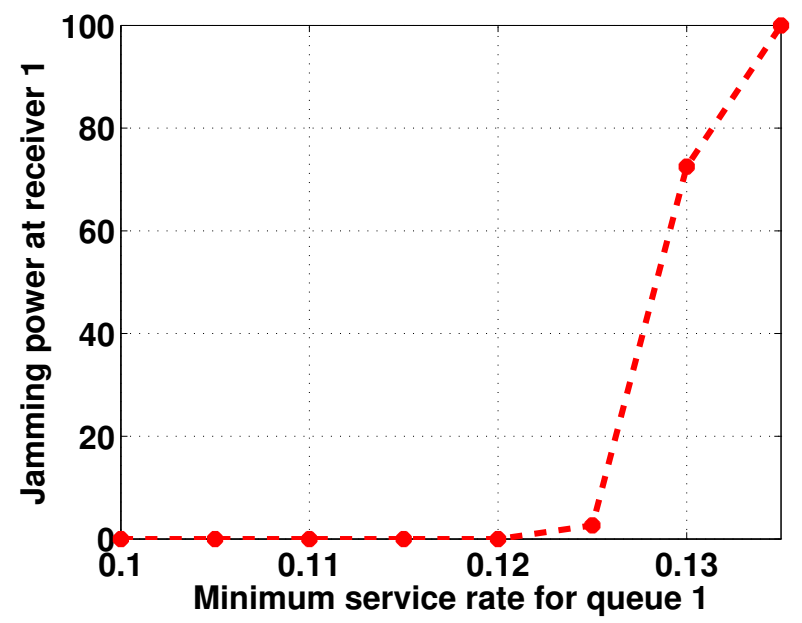

Fig. 13. Power of the jamming signal sent by receiver $1\left(P_{J}\right)$ vs Minimum service rate $\left(\mu_{t h}\right): P_{1}=P_{2}=100, P_{J}=100, \alpha=2.3, d=10, c=1.1$, $d_{1}=d, d_{2}=c d, d_{3}=c d, \gamma_{1}=0.5, \gamma_{2}=0.4, \lambda_{1}=0.05$, and $\beta=10 d B$.

perform successive decoding, the role of jamming signal to ensure secrecy of the packets intended to receiver 1 is more crucial. It is also found that performing power control at the transmitter or receiver 1 can enlarge the stability region significantly. When the self-interference cancelation is not efficient, performing power control at receiver 1 can help to increase the stability throughput significantly. From the optimization problem considered in this paper, it is found that the jamming capability of receiver 1 can help to guarantee minimum service rate for the first queue and maximize the saturated throughput of receiver 2 by appropriately choosing the jamming power.

\section{REFERENCES}

[1] P. Mohapatra, N. Pappas, J. Lee, T. Q. S. Quek, and V. Angelakis, "Stability region of two-user full-duplex broadcast channel with secrecy constraint," in Proc. IEEE Int. Conf. on Communications, May 2016. 
[2] R. Rao and A. Ephremides, "On the stability of interacting queues in a multiple-access system," IEEE Trans. Inf. Theory, vol. 34, no. 5, pp. 918-930, Sep. 1988.

[3] T. Cover, "An achievable rate region for the broadcast channel," IEEE Trans. Inf. Theory, vol. 21, no. 4, pp. 399-404, Jul. 1975.

[4] K. Marton, "A coding theorem for the discrete memoryless broadcast channel," IEEE Trans. Inf. Theory, vol. 25, no. 3, pp. 306-311, May 1979.

[5] A. Jafarian and S. Vishwanath, "The two-user Gaussian fading broadcast channel," in Proc. IEEE Int. Symp. Inf. Theory, Jul. 2011, pp. 2964 2968.

[6] I. Csiszar and J. Korner, "Broadcast channels with confidential messages," IEEE Trans. Inf. Theory, vol. 24, no. 3, pp. 339-348, May 1978.

[7] E. Ekrem and S. Ulukus, "Secrecy in cooperative relay broadcast channels," IEEE Trans. Inf. Theory, vol. 57, no. 1, pp. 137-155, Jan. 2011.

[8] A. Wyner, "The wire-tap channel," Bell Syst. Tech. J., vol. 54, no. 8, pp. 1334-1387, Oct. 1975.

[9] A. Khisti, A. Tchamkerten, and G. W. Wornell, "Secure broadcasting over fading channels," IEEE Transactions on Information Theory, vol. 54, no. 6, pp. 2453-2469, June 2008.

[10] R. Liu, I. Maric, P. Spasojević, and R. Yates, "Discrete memoryless interference and broadcast channels with confidential messages: Secrecy rate regions," IEEE Trans. Inf. Theory, vol. 54, no. 6, pp. 2493-2507, Jun. 2008.

[11] O. Koyluoglu and H. El Gamal, "Cooperative encoding for secrecy in interference channels," IEEE Trans. Inf. Theory, vol. 57, no. 9, pp. 56825694, Sep. 2011.

[12] E. Ekrem and S. Ulukus, "Effects of cooperation on the secrecy of multiple access channels with generalized feedback," in Proc. CISS, Mar. 2008, pp. 791-796.

[13] J. Barros and M. Rodrigues, "Secrecy capacity of wireless channels," in Proc. IEEE Int. Symp. Inf. Theory, Jul. 2006, pp. 356-360.

[14] A. Ephremides and B. Hajek, "Information theory and communication networks: an unconsummated union," IEEE Trans. Inf. Theory, vol. 44, no. 6, pp. 2416-2434, Oct. 1998.

[15] G. Fayolle, E. Gelenbe, and J. Labetoulle, "Stability and optimal control of the packet switching broadcast channel," J. ACM, vol. 24, no. 3, pp. 375-386, Jul. 1977.

[16] N. Pappas, M. Kountouris, A. Ephremides, and V. Angelakis, "Stable throughput region of the two-user broadcast channel," CoRR, vol. abs/1604.08418, 2016.

[17] N. Pappas and M. Kountouris, "The stability region of the two-user broadcast channel," in Proc. IEEE Int. Conf. on Communications, May 2016.

[18] N. Pappas, M. Kountouris, and A. Ephremides, "The stability region of the two-user interference channel," in Proc. IEEE Inf. Theory Workshop, Sept 2013, pp. 1-5.

[19] S. Kompella and A. Ephremides, "Stable throughput regions in wireless networks," Foundations and Trends in Networking, vol. 7, no. 4, pp. 235338, 2014. [Online]. Available: http://dx.doi.org/10.1561/1300000039

[20] Y. Sarikaya and O. Ercetin, "On physically secure and stable slotted aloha system," in Proc. 47th Annu. Allerton Conf. Commun., Control, Comput., Sept. 2009, pp. 1090-1096.

[21] Y. Liang, H. V. Poor, and L. Ying, "Wireless broadcast networks: Reliability, security, and stability," in Information Theory and Applications Workshop, Jan 2008, pp. 249-255.

[22] _ _ "Secure communications over wireless broadcast networks: Stability and utility maximization," IEEE Trans. Inf. Forensics and Security, vol. 6, no. 3, pp. 682-692, Sept 2011.

[23] G. Zheng, I. Krikidis, J. Li, A. P. Petropulu, and B. Ottersten, "Improving physical layer secrecy using full-duplex jamming receivers," IEEE Transactions on Signal Processing, vol. 61, no. 20, pp. 4962-4974, Oct 2013.

[24] G. Chen, Y. Gong, P. Xiao, and J. A. Chambers, "Physical layer network security in the full-duplex relay system," IEEE Transactions on Information Forensics and Security, vol. 10, no. 3, pp. 574-583, March 2015.

[25] B. Akgun, O. O. Koyluoglu, and M. Krunz, "Exploiting full-duplex receivers for achieving secret communications in multiuser MISO networks," IEEE Transactions on Communications, vol. 65, no. 2, pp. 956968, Feb 2017.

[26] B. He, X. Zhou, and A. L. Swindlehurst, "On secrecy metrics for physical layer security over quasi-static fading channels," IEEE Transactions on Wireless Communications, vol. 15, no. 10, pp. 6913-6924, Oct 2016.
[27] E. Gvenkaya, J. M. Hamamreh, and H. Arslan, "On physical-layer concepts and metrics in secure signal transmission," Physical Communication, vol. 25, no. Part 1, pp. 14 - 25, 2017. [Online]. Available: http://www.sciencedirect.com/science/article/pii/S1874490717300903

[28] D. W. K. Ng, E. S. Lo, and R. Schober, "Dynamic resource allocation in mimo-ofdma systems with full-duplex and hybrid relaying," IEEE Transactions on Communications, vol. 60, no. 5, pp. 1291-1304, May 2012.

[29] T. Riihonen, S. Werner, and R. Wichman, "Mitigation of loopback selfinterference in full-duplex mimo relays," IEEE Transactions on Signal Processing, vol. 59, no. 12, pp. 5983-5993, Dec 2011.

[30] D. Kim, H. Ju, S. Park, and D. Hong, "Effects of channel estimation error on full-duplex two-way networks," IEEE Transactions on Vehicular Technology, vol. 62, no. 9, pp. 4666-4672, Nov 2013.

[31] A. C. Cirik, Y. Rong, and Y. Hua, "Achievable rates of full-duplex mimo radios in fast fading channels with imperfect channel estimation," IEEE Transactions on Signal Processing, vol. 62, no. 15, pp. 3874-3886, Aug 2014.

[32] J. Lee and T. Q. S. Quek, "Hybrid full-/half-duplex system analysis in heterogeneous wireless networks," IEEE Transactions on Wireless Communications, vol. 14, no. 5, pp. 2883-2895, May 2015.

[33] Z. Tong and M. Haenggi, "Throughput analysis for full-duplex wireless networks with imperfect self-interference cancellation," IEEE Transactions on Communications, vol. 63, no. 11, pp. 4490-4500, Nov 2015.

[34] N. Pappas, M. Kountouris, A. Ephremides, and A. Traganitis, "Relayassisted multiple access with full-duplex multi-packet reception," IEEE Trans. on Wir. Comm., vol. 14, no. 7, pp. 3544-3558, July 2015.

[35] I. Avgouleas, N. Pappas, D. Yuan, and V. Angelakis, "Probabilistic cooperation of a full-duplex relay in random access networks," IEEE Access, vol. 5, pp. 7394-7404, 2017.

[36] W. Szpankowski, "Stability conditions for some distributed systems: Buffered random access systems," Advances in Applied Probability, pp. 498-515, 1994.

[37] A. K. Sadek, K. J. R. Liu, and A. Ephremides, "Cognitive multiple access via cooperation: Protocol design and performance analysis," IEEE Transactions on Information Theory, vol. 53, no. 10, pp. 3677-3696, Oct 2007.

[38] S. Sarma, S. Shukla, and J. Kuri, "Joint scheduling \& jamming for data secrecy in wireless networks," in Proc. Int. Symp. Modeling Optimization Mobile, May 2013, pp. 248-255.

[39] S. Bashar and Z. Ding, "Optimum power allocation against information leakage in wireless network," in Proc. IEEE Global Telecommunications Conference, Nov 2009, pp. 1-6.

[40] — "Optimum routing protection against cumulative eavesdropping in multihop wireless networks," in Proc. IEEE Military Communications Conference, Oct 2009, pp. 1-7.

[41] C. Capar, D. Goeckel, B. Liu, and D. Towsley, "Secret communication in large wireless networks without eavesdropper location information," in Proc. IEEE INFOCOM, March 2012, pp. 1152-1160.

[42] J. Lee, A. Conti, A. Rabbachin, and M. Z. Win, "Distributed network secrecy," IEEE Journal on Selected Areas in Communications, vol. 31, no. 9, pp. 1889-1900, Sep 2013.

[43] D. Klinc, J. Ha, S. W. McLaughlin, J. Barros, and B. J. Kwak, "Ldpc codes for the gaussian wiretap channel," IEEE Transactions on Information Forensics and Security, vol. 6, no. 3, pp. 532-540, Sept 2011.

[44] W. Yang, R. F. Schaefer, and H. V. Poor, "Finite-blocklength bounds for wiretap channels," in 2016 IEEE International Symposium on Information Theory (ISIT), July 2016, pp. 3087-3091.

[45] —, "Wiretap channels: Nonasymptotic fundamental limits," CoRR, vol. abs/1706.03866, 2017. [Online]. Available: http://arxiv.org/abs/1706.03866

[46] A. El Gamal and Y.-H. Kim, Network information theory. Cambridge university press, 2011.

[47] A. Sengupta, R. Tandon, and T. C. Clancy, "Fundamental limits of caching with secure delivery," IEEE Transactions on Information Forensics and Security, vol. 10, no. 2, pp. 355-370, Feb 2015.

[48] M. A. Maddah-Ali and U. Niesen, "Fundamental limits of caching," IEEE Transactions on Information Theory, vol. 60, no. 5, pp. 28562867, May 2014.

[49] N. Pappas, Z. Chen, and I. Dimitriou, "Throughput and delay analysis of wireless caching helper systems with random availability," IEEE Access, vol. 6, pp. 9667-9678, 2018. 


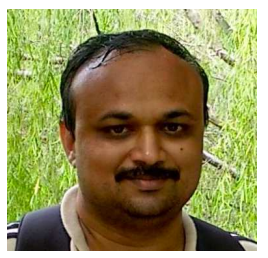

Parthajit Mohapatra received the B.E. degree in electronics and communication engineering, from Biju Patnaik University of Technology, Odisha, India, in 2003 and the M.Tech. degree in electronic systems and communications from the National Institute of Technology, Rourkela, India, in 2006. He obtained his $\mathrm{Ph}$. D. degree in electrical communication engineering from Indian Institute of science, Bangalore, India, in 2015. He was working as a postdoctoral research fellow at iTrust, center for research in cyber security at Singapore University of Technology and Design, Singapore, during 2015-2016.

$\mathrm{He}$ is currently working as an assistant professor at G. S. Sanyal School of Telecommunications at Indian Institute of Technology, Kharagpur, India. His research interests are in the areas of information theoretic secrecy, advanced communication techniques for wireless communication, and union ory.

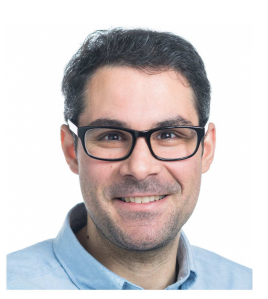

Nikolaos Pappas (S'07-M'13) received the B.Sc. degree in computer science, the B.Sc. degree in mathematics, the M.Sc. degree in computer science, and the Ph.D. degree in computer science from the University of Crete, Greece, in 2005, 2012, 2007 , and 2012, respectively. He is currently an Associate Professor of mobile telecommunications with the Department of Science and Technology, Linköping University, Norrköping, Sweden. From 2005 to 2012, he was a graduate Research Assistant with the Telecommunications and Networks Laboratory, Institute of Computer Science, Foundation for Research and Technology, Hellas, and a Visiting Scholar with the Institute of Systems Research, University of Maryland at College Park. From 2012 to 2014, he was a Post-Doctoral Researcher with the Department of Telecommunications, Supélec, France. Since 2014, he has been with the University of Linköping, Norrköping, as a Marie Curie Fellow (IAPP). His main research interests are in the field of wireless communication networks with emphasis on the stability analysis, energy harvesting networks, network-level cooperation, ageof-information, network coding, and stochastic geometry. From 2013 to 2018 he was Editor of the IEEE Communications Letters.

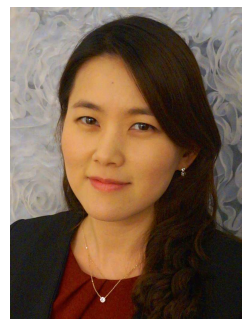

Jemin Lee (S'06-M'11) received the B.S. (with high honors), M.S., and Ph.D. degrees in Electrical and Electronic Engineering from Yonsei University, Seoul, Korea, in 2004, 2007, and 2010, respectively. She was a Postdoctoral Fellow at the Massachusetts Institute of Technology (MIT), Cambridge, MA from 2010 to 2013, and a Temasek Research Fellow at iTrust, Centre for Research in Cyber Security, Singapore University of Technology and Design (SUTD), Singapore from 2014 to 2016. Currently, she is an Assistant Professor at the Department of Information and Communication Engineering, Daegu Gyeongbuk Institute of Science and Technology (DGIST), Daegu, Korea. Her current research interests include wireless communications, wireless security, intelligent networking, and machine-type communications.

Dr. Lee is currently an Editor for the IEEE TRANSACTIONS ON WIRELESS COMMUNICATIONS and the IEEE COMMUNICATIONS LETTERS, and served as a Guest Editor for the IEEE WIRELESS COMMUNICATIONS, special issue on LTE in Unlicensed Spectrum, 2016, and the ELSEVIER PHYSICAL Communication, special issues on Physical Layer Security in 2016 and Heterogeneous and Small Cell Networks in 2014. She received the IEEE ComSoc AP Outstanding Paper Award in 2017, the IEEE ComSoc AP Outstanding Young Researcher Award in 2014, the Temasek Research Fellowship in 2013, and the Chun-Gang Outstanding Research Award in 2011.

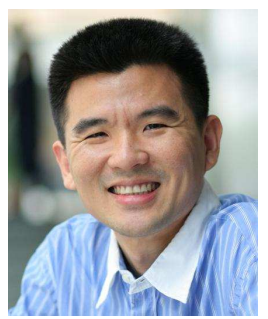

Tony Q.S. Quek (S'98-M'08-SM'12-F'18) received the B.E. and M.E. degrees in Electrical and Electronics Engineering from Tokyo Institute of Technology, respectively. At MIT, he earned the Ph.D. in Electrical Engineering and Computer Science. Currently, he is a tenured Associate Professor with the Singapore University of Technology and Design (SUTD). He also serves as the Associate Head of ISTD Pillar and the Deputy Director of the SUTD-ZJU IDEA. His current research topics include wireless communications and networking, internet-of-things, network intelligence, wireless security, and big data processing.

Dr. Quek has been actively involved in organizing and chairing sessions, and has served as a member of the Technical Program Committee as well as symposium chairs in a number of international conferences. He is currently an elected member of IEEE Signal Processing Society SPCOM Technical Committee. He was an Executive Editorial Committee Member for the IEEE TRANSACTIONS ON WiRELESS COMMUNiCATIONS, an Editor for the IEEE TRANSACTIONS ON COMMUNiCATIONS, and an Editor for the IEEE WiRELESS COMMUNiCATIONS LETTERS. He is a co-author of the book "Small Cell Networks: Deployment, PHY Techniques, and Resource Allocation" published by Cambridge University Press in 2013 and the book "Cloud Radio Access Networks: Principles, Technologies, and Applications" by Cambridge University Press in 2017.

Dr. Quek was honored with the 2008 Philip Yeo Prize for Outstanding Achievement in Research, the IEEE Globecom 2010 Best Paper Award, the 2012 IEEE William R. Bennett Prize, the 2015 SUTD Outstanding Education Awards - Excellence in Research, the 2016 IEEE Signal Processing Society Young Author Best Paper Award, the 2017 CTTC Early Achievement Award, the 2017 IEEE ComSoc AP Outstanding Paper Award, and the 2017 Clarivate Analytics Highly Cited Researcher. He is a Distinguished Lecturer of the IEEE Communications Society and a Fellow of IEEE.

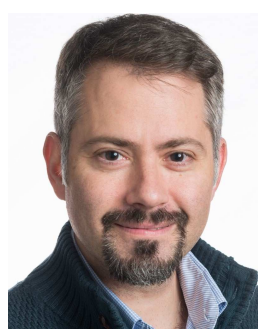

Vangelis is an associate professor with the Dept. of Science and Technology (ITN), Linköping University, Norrköping, Sweden. In 2015 he received his Docent from ITN-LiU. He holds a PhD from the Dept. of Computer Science of the University of Crete, Greece, acquired in 2008. His research interests revolve around design of telecommunication systems' and networks' resources optimization with a focus on IoT and Smart City applications. He has held multiple guest posts both in the industry and academia, primarily through EU funded projects, in US, EU, and China. Vangelis is coordinating the EU FP7 MSCA IAPP project (SOrBet), and is a principal investigator for $\mathrm{LiU}$ in the EU FP7 SMARTCITIES RERUM project and the EU Horizon 2020 MSCA ITN WiVi2020 project. He is also been acting as project manager within two more EUfunded projects coordinated by the Mobile Telecommunications group and has been in the OPTICWISE COST Action management committee.

Vangelis has more than 60 papers in international journals and peerreviewed conferences. He is currently editing a Springer book in Smart Cities, due in 2016, and co-organizing the IEEE Globecom'15 workshop in Optimizing Heterogeneous Networking Technologies for the Internet of Things. Vangelis serves as an associate editor of the IEEE/KICS journal of communications and networks (JCN), and he has been serving as organizer and technical program committee member in a wide range of international conferences and workshops. 